ज्ञ FRANÇAISE

$>\mathrm{DE}$

딜 PEAGOGIE

\section{Revue française de pédagogie}

Recherches en éducation

172 | juillet-septembre 2010

La pédagogie universitaire : un courant en plein développement

\title{
Fonctions, structuration et évolution des croyances (et connaissances) des enseignants
}

The functions, structures and changes of the teachers' beliefs (and knowledge) Funciones, estructuración y evolución de las creencias (y conocimientos) de los docentes

Funktion, Strukturierung und Entwicklung der Vorstellungen (und der Kenntnisse) der Lehrer

Marcel Crahay, Philippe Wanlin, Élisabeth Issaieva et Isabelle Laduron

\section{OpenEdition}

\section{Édition électronique}

URL : http://journals.openedition.org/rfp/2296

DOI : $10.4000 / \mathrm{rfp} .2296$

ISSN : 2105-2913

\section{Éditeur}

ENS Éditions

\section{Édition imprimée}

Date de publication : 15 juillet 2010

Pagination : 85-129

ISBN : 978-2-7342-1188-4

ISSN : 0556-7807

Référence électronique

Marcel Crahay, Philippe Wanlin, Élisabeth Issaieva et Isabelle Laduron, « Fonctions, structuration et évolution des croyances (et connaissances) des enseignants », Revue française de pédagogie [En ligne], 172 | juillet-septembre 2010, mis en ligne le 01 décembre 2014, consulté le 01 mai 2019. URL : http:// journals.openedition.org/rfp/2296; DOI : 10.4000/rfp.2296 


\section{NOTE DE SYNTHÈSE}

\section{Fonctions, structuration et évolution des croyances (et connaissances) des enseignants Marcel Crahay, Philippe Wanlin, Élisabeth Issaieva et Isabelle Laduron}

Les recherches relatives aux croyances et connaissances des enseignants constituent un ensemble assez disparate. La définition même du concept de croyance ainsi que la distinction entre croyance et connaissance sont objets de controverse. En revanche, à la question des catégories de croyances et de connaissances mobilisées par les enseignants dans le cadre de leur métier correspondent des réponses plutôt convergentes. Après avoir résumé les contributions majeures concernant ces thématiques, la présente note de synthèse aborde une problématique cruciale eu égard à la formation des enseignants : les croyances et connaissances des enseignants évoluent-elles et si oui, dans quelles conditions ? Plusieurs courants de recherche sont examinés dans cette perspective : les recherches portant sur les changements dits "naturels ", un ensemble auquel nous adjoignons celles portant sur les stades de développement ponctuant la carrière des enseignants; les études s'attachant à mesurer les effets des programmes de formation des enseignants dont la plupart concernent la formation initiale; les recherches relevant du courant nommé staff development. Une section porte sur les croyances des enseignants débutants, car les recherches menées à ce sujet permettent de mieux comprendre les études évaluant les effets des programmes de formation. La discussion finale est articulée autour du constat assez général de la grande difficulté à faire évoluer les croyances des enseignants. Nous détaillons diverses hypothèses qui ont été avancées pour tenter d'expliquer la relative imperméabilité de ces croyances aux processus de formation. Nous soulevons ensuite une question, à nos yeux essentielle et pourtant rarement débattue : existe-t-il de meilleures croyances que d'autres ? Enfin nous terminons par une discussion de l'apport de Leuchter qui permet de recadrer la problématique des changements qui s'opèrent au niveau des croyances et connaissances des enseignants.

Descripteurs (TESE) : savoirs, développement des capacités professionnelles, enseignant stagiaire, pratique pédagogique, formation des enseignants.

\section{INTRODUCTION}

La majorité des études empiriques portant sur ce que pensent les enseignants de l'enseignement et de l'apprentissage sont publiées dans des revues anglophones. Dans cette sphère culturelle, le terme de belief (croyance) s'est 
imposé alors que, dans la littérature francophone, le terme de "représentation » est privilégié. En fait, à bien y regarder, qu'elle soit anglophone ou francophone, la littérature de recherche abonde en termes différents: théories personnelles, perspectives, conceptions, préconceptions, théories implicites, perceptions, attitudes, dispositions... Bien que ces termes aient des définitions différentes, ils sont difficilement différenciables. Dans sa revue de la littérature, Pajares (1992) les considère d'ailleurs comme interchangeables (1).

La difficulté de définir le concept de croyance est largement reconnue. Discutant ce problème, Pajares (1992) mentionne plusieurs définitions ; il en retient deux qui, tout en étant complémentaires, lui paraissent prototypiques de deux tendances différentes, décelables dans l'ensemble des productions des chercheurs. La première définition renvoie à " toute proposition simple, consciente ou inconsciente, inférée à partir de ce qu'une personne dit ou fait, pouvant être précédé par la phrase "je crois que..." " (Rokeach, 1976, cité par Pajares, 1992, p. 314). La deuxième voit la croyance comme " une représentation que se fait un individu de la réalité ; celle-ci possède assez de validité, de vérité, ou de crédibilité pour guider la pensée et le comportement » (Harvey, 1986, cité par Pajares, 1992, p. 313). Si la première définition est strictement opérationnelle (2), la deuxième renvoie à l'idée d'un « quelque chose » d'ordre cognitif mobilisé par les enseignants dans certaines circonstances, et qui véhicule un contenu ayant trait à l'expérience de l'enseignement. On peut considérer que cette propriété - un contenu relatif à l'enseignement - constitue le noyau dur, faisant donc consensus, de toutes les recherches regroupées sous le label «teacher's beliefs (3)». À ce stade de la réflexion, on peut donc considérer que le concept de croyances des enseignants renvoie à des contenus mentaux, ayant trait à l'enseignement, compilés dans des schémas ou des concepts, pouvant prendre la forme de propositions ou d'assertions.

Certains auteurs (Fenstermacher, 1979 ; Hofer \& Pintrich, 1997 ; Nespor, 1987) ont tenté de clarifier la distinction entre croyances (beliefs) et connaissances (knowledge), notamment pour montrer qu'il y a une différence de statut épistémique entre les deux termes. Les connaissances supposent un accord entre les esprits - un accord intersubjectif - fondé sur un ensemble de preuves et/ou d'arguments qui permettent de justifier la validité de la (ou des) proposition(s) retenue(s) ou, au moins, sa (ou leur) plus grande vraisemblance par rapport à d'autres conceptions relatives au même objet ou au même phénomène. Par extension, on peut considérer qu'en principe, les croyances ne présupposent pas d'accord intersubjectif reposant sur un système de validation rigoureux (4). Certes bien des croyances sont largement partagées au sein de certains groupes sociaux, mais leur évidence tient davantage au fait même qu'elles sont répandues et non contestées qu'au fait qu'elles ont été prouvées ou justifiées par une argumentation rigoureuse. Si la majorité des croyances sont d'origine sociale, elles impliquent une adhésion individuelle que des enseignants signalent, par exemple, en affirmant: "Je sais que des recherches concluent à l'effet négatif du redoublement mais, moi, je crois que le redoublement peut parfois servir " (Marcoux \& Crahay, 2008). Ainsi les croyances des enseignants engloberaient à la fois des éléments personnels et des éléments partagés par l'ensemble de la communauté enseignante (Verloop, Van Driel \& Meijer, 2001). Elles sont à considérer comme une caractéristique psychologique de l'individu, tout en étant enracinées dans son substrat culturel. Ceci permet, selon nous, de rapprocher ce concept de celui de représentation et, plus fondamentalement, de considérer les croyances et les représentations comme des constructions à la fois cognitives et sociales. Autrement dit, les croyances ou encore les représentations des enseignants ont une double nature, indissociablement individuelle et sociale. Ceci ne signifie pas 
pour autant que tous les enseignants partagent des croyances identiques, ni qu'ils agissent de commune façon en accord avec un corpus indifférencié de croyances. Des recherches (Halkes \& Deijkers, 2003 ; Munby, 1983) dévoilent, de la part d'enseignants différents, un recours idiosyncrasique aux connaissances et croyances pour sélectionner les actes d'enseignement, contrôler leur processus et évaluer leurs effets. D'autres recherches indiquent le recours à des orientations professionnelles ou à des profils de croyances ou de cognitions différents selon les enseignants, aboutissant à des comportements instructionnels hétérogènes (Buchmann, 1987, 1986).

Dans la littérature, il apparaît également que les frontières entre connaissances et croyances sont difficilement identifiables, ce qui conduit bon nombre de spécialistes à traiter ces deux termes de manière concomitante (Borko \& Putnam, 1996 ; Calderhead, 1996 ; Fenstermacher, 1994 ; Pajares, 1992 ; Vause, 2009 ; Woolfolk Hoy, Davis \& Pape, 2006), voire à estimer qu'il serait plus judicieux de considérer les savoirs enseignants comme étant essentiellement des croyances (Kagan, 1992). On retiendra essentiellement de ces travaux que les connaissances feraient davantage référence à des éléments factuels ou empiriques, alors que les croyances renverraient plutôt à des suppositions ou idéologies (5), les unes et les autres étant fortement empreintes des expériences personnelles et collectives des enseignants.

Dans un article riche en réflexions, Kagan (1992) propose de considérer les croyances des enseignants comme une forme de connaissance personnelle, composée de conjectures, le plus souvent implicites, concernant les élèves, l'apprentissage, la classe, le processus d'enseignement et les objets à enseigner (6). Pour cette auteure, qui reprend à son compte les thèses de Broudy (1980), de Floden et Clark (1988) et de Lieberman (1992), il convient de reconnaître que l'enseignement est un domaine caractérisé par l'absence quasi totale de vérités, de certitudes ou de connaissances scientifiquement établies sur la bonne façon d'enseigner (7). II est donc normal que les enseignants se dotent d'une pédagogie personnelle (personal ou personalized pedagogy, dans le texte). Celle-ci mélange des éléments tirés de l'expérience propre de l'enseignant, d'autres inspirés du sens commun de la communauté enseignante ou, plus largement, de la communauté culturelle à laquelle il appartient, et d'autres encore venant de théories scientifiques, psychologiques, linguistiques, etc. Dans cette perspective, les connaissances constitueraient un sous-ensemble des croyances des enseignants, sous-ensemble caractérisé par le fait qu'il regroupe des assertions soutenues par des preuves empiriques et une argumentation logique. Pour l'essentiel, les auteurs de la présente note de synthèse partagent le point de vue de Kagan (1992): dès lors que nous utiliserons l'expression "croyances des enseignants ", il faut sous-entendre le terme " connaissances".

Pour certains chercheurs (cf. en particulier Calderhead, 1996), les connaissances et les croyances des enseignants sont en quelque sorte "encapsulées " dans leurs expériences professionnelles, au point qu'elles en sont indissociables. Elles se manifestent au travers des actes d'enseignement, qu'il s'agisse de planification d'activité, d'interactions avec les élèves ou d'évaluation. Elles s'expriment sous forme de métaphores (8), d'actions ou de paroles. Leur nature relève autant de l'image mentale que d'idées ou de propositions, implicites ou explicites, conscientes ou inconscientes, inférées à partir de ce que les enseignants disent ou font, celles-ci pouvant être précédées par la phrase "Je crois que... ", dans le cas où elles sont de nature déclarative. Ces images, idées ou propositions sont supposées avoir, du point de vue de l'enseignant qui en est porteur, assez de validité ou de crédibilité pour orienter ses actions, de même que certains de ses 
jugements et décisions. De façon largement consensuelle (cf. notamment Borko \& Shavelson, 1990 ; Calderhead, 1996 ; Woolfolk Hoy, Davis \& Pape, 2006), les chercheurs considèrent que les connaissances et les croyances des enseignants, inévitablement modelées par leurs expériences personnelles et professionnelles, servent de filtres pour analyser et gérer les situations d'enseignement. Ainsi, par exemple, le curriculum officiel ne serait jamais saisi ni implémenté tel qu'il est écrit ; son influence sur la pratique des enseignants serait toujours médiatisée à travers les filtres que constituent entre autres leurs connaissances et croyances pédagogiques générales, disciplinaires et pédagogiques de contenu, leur expérience personnelle et professionnelle, leurs représentations ainsi que leurs perceptions relatives aux élèves (Remillard, 2005). Dans la foulée, nombreux sont ceux qui postulent une influence des croyances sur les pratiques d'enseignement (Bandura, 1986 ; Dewey, 1933 ; Fenstermacher, 1979 ; Huber \& Roth, 1990 ; Kremer, 1981 ; Munby, 1982, 1983 ; Nisbett \& Ross, 1980 ; Pajares, 1992 ; Rokeach, 1976 ; Staub \& Stern, 2002 ; Stipek, Givvin, Salmon et al., 2001 ; Tabachnick \& Zeichner, 1984). Cette influence est pourtant loin d'être prouvée de façon absolue, ce qui a conduit certaines auteurs (cf. notamment Hancock \& Gallard, 2004 ; Mellado, 1998) à douter radicalement de la liaison entre croyances et pratiques. Notons, pour être complet, que des résultats entre ces deux extrêmes ont également été mis en évidence par la recherche: les croyances et les connaissances des enseignants entretiendraient un lien conditionnel avec leurs pratiques (Baumert \& Kunter, 2006 ; Calderhead, 1996 ; Diedrich, Thussbas \& Klieme, 2002 ; Klieme \& Reusser, 2003 ; Leuchter, 2009 ; Leuchter, Reusser, Pauli et al., 2008 ; Lipowsky, 2006 ; Opdenakker \& Van Damme, 2006).

Bien qu'importante, cette question du lien entre croyances et pratiques d'enseignement n'est pas au centre de la présente note de synthèse. Son objet principal concerne les types de croyances et connaissances des enseignants, leur organisation ainsi que leur développement au travers de la formation initiale et continuée des enseignants, de même qu'au long de leur carrière professionnelle.

\section{CATÉGORIES DE CROYANCES ET DE CONNAISSANCES DES ENSEIGNANTS}

La littérature distingue plusieurs types de connaissances ou croyances (Calderhead, 1996 ; Leuchter, 2009 ; Shulman, 1987 ; Woolfolk Hoy, Davis \& Pape, 2006). Nous devons la première typologie à Shulman (1987) qui distingue sept types de connaissances :

- les connaissances disciplinaires (content knowledge) qui correspondent aux savoirs que les enseignants ont de la matière qu'ils enseignent ;

- les connaissances pédagogiques générales (general pedagogical knowledge) incluant les principes et stratégies de gestion de groupe et d'organisation, notamment des interactions, qui transcendent les matières scolaires ;

- les connaissances curriculaires (curriculum knowledge) avec une centration particulière sur le matériel, les manuels scolaires et le programme qui servent de base pour l'enseignement ;

- les connaissances pédagogiques du contenu (pedagogical content knowledge) qui sont un amalgame, spécifique à chaque enseignant, articulant connaissances pédagogiques et disciplinaires ; il s'agit, par exemple, du savoir qu'a construit l'enseignant du primaire en ce qui concerne la façon d'enseigner les fractions à des élèves de 10 ans ;

- les connaissances relatives aux apprenants et à leurs caractéristiques (knowledge of learners and their characteristics) qui renvoient, d'une part, aux savoirs sur les élèves en général et, d'autre part, sur des élèves spécifiques de 
la classe. La psychologie de l'apprentissage et du développement sont des éléments centraux de ces connaissances ;

- les connaissances relatives au contexte éducatif, au système scolaire et à la société (knowledge of educational context) ;

- les connaissances relatives aux finalités de l'éducation (knowledge of educational ends), ses buts, ses valeurs et leurs bases philosophiques et historiques.

L'architecture simplifiée de Borko et Putnam (1996) refond les sept types initialement proposés par Shulman (1987) en une typologie faite de trois ensembles (9) :

- les croyances et connaissances pédagogiques générales (general pedagogical knowledge and beliefs) : ce sont les savoirs et conceptions de l'enseignant concernant l'enseignement, l'apprentissage et les apprenants, qui transcendent les disciplines scolaires. Elles intègrent les connaissances et croyances des enseignants relatives à la gestion de la classe (comment faire travailler ensemble un groupe d'élèves et maintenir son engagement dans les activités), aux stratégies instructionnelles (comment piloter une leçon, comment créer, planifier et structurer des environnements éducatifs, quelles stratégies ou routines pédagogiques ou interactionnelles utiliser parmi le répertoire professionnel, etc.) ainsi qu'aux apprenants, à leur apprentissage et à la manière de leur enseigner (comment les élèves pensent-ils et intègrent-ils la matière, comment l'enseignant peut-il contribuer à cet apprentissage). À noter que la recherche montre que ces connaissances ou croyances relatives aux élèves, qui occupent une grande part du répertoire cognitif des enseignants, sont d'une grande importance dans leur agir efficace (Marland, 1993 ; Mayer \& Marland, 1997). Ce type de connaissances ou croyances renferme également les éléments cognitifs renvoyant aux programmes, au système éducatif, à la société, etc. ;

- les croyances et connaissances disciplinaires (subject matter knowledge and beliefs) : ce sont les conceptions et savoirs des enseignants dans une matière spécifique. Ils rassemblent les connaissances et croyances concernant les faits, les concepts et la terminologie d'une discipline particulière ainsi que ses idées organisatrices, les connexions entre ces idées, les réflexions et arguments qui la fondent, son développement, etc. ;

- les croyances et connaissances pédagogiques du contenu (pedagogical content knowledge and beliefs) : il s'agit de la traduction des connaissances et croyances disciplinaires médiatisée par les connaissances et croyances pédagogiques générales pour en favoriser l'enseignement. Elles englobent les savoirs et les conceptions sur les manières de présenter et de formuler la matière disciplinaire pour la rendre compréhensible à des tiers, ainsi qu'une compréhension de ce qui rend complexe ou simple l'apprentissage de certains de ses éléments.

S'inspirant du modèle écologique de Bronfenbrenner (1986), Woolfolk Hoy, Davis et Pape (2006) proposent un modèle en forme de poupées russes. Au niveau le plus large, ils distinguent les croyances et connaissances des enseignants eu égard à l'enfance ou à l'adolescence et le sens donné par ceux-ci à la diversité des individus. À un second niveau, inclus dans le précédent, il y a les croyances et connaissances des enseignants en ce qui concerne le contexte politique de l'éducation (les réformes, les standards, les idées de responsabilité et les politiques de pilotage, etc.). Au niveau suivant, se situeraient les croyances et connaissances en rapport avec le contexte immédiat de la classe : les caractéristiques des élèves (notamment, le genre, la classe sociale, etc.), le contenu d'enseignement, etc. 
Enfin, au centre du modèle, Woolfolk Hoy, Davis et Pape (2006) placent ce qui a trait à l'identité professionnelle et au sentiment d'efficacité (self efficacy). Cette dernière strate de croyances et connaissances serait cruciale pour l'adaptabilité des enseignants aux situations mouvantes et souvent conflictuelles qu'ils doivent gérer.

\section{MULTIDIMENSIONNALITÉ ET MULTIFONCTIONNALITÉ DES CROYANCES}

Comme déjà mentionné, les débats actuels dans la littérature anglo-saxonne s'éloignent des discussions conceptuelles opposant croyances et connaissances pour préférer un vocabulaire rassembleur: teachers' beliefs and knowledge pour les uns (cf. notamment Woolfolk Hoy, Davis \& Pape, 2006) ou teachers' capacity pour d'autres (Grant, 2008 ; Grant \& Agosto, 2008 ; Howard \& Aleman, 2008 ; McDiarmid \& Clevenger-Bright, 2008), indiquant par là le caractère hétérogène des structures mentales mobilisées par les enseignants. Sous des terminologies différentes (10), les chercheurs convergent vers l'idée que les recherches futures doivent cibler la compréhension profonde du fonctionnement des croyances et connaissances des enseignants sur le lien entre les croyances et connaissances disciplinaires et les pedagogical content knowledge, sur leur utilisation flexible dans l'action, une même croyance pouvant, comme le notent Richardson et Placier (2001), se traduire en des actions différentes selon la situation et le type d'élève.

Leuchter (2009) estime que les connaissances et croyances des enseignants sont soumises continuellement à une pression temporelle et à des situations problématiques complexes. Utilisant les apports des recherches sur le savoir expert des enseignants, elle propose de caractériser leurs croyances et connaissances en les positionnant sur quatre continuums allant du systématique au situé, de l'explicite à l'implicite, du déclaratif au procéduralisé, et du scientifiquement fondé aux éléments basés sur l'expérience personnelle et professionnelle. Toujours selon la conceptualisation de cette chercheuse - un apport sur lequel nous reviendrons dans la discussion -, l'agir professionnel des enseignants englobe des connaissances et des croyances mises en réseau, celles-ci naviguant en quelque sorte dans l'espace défini par ces quatre dimensions. Ces combinaisons de croyances et connaissances déterminent, avec d'autres facteurs liés au contexte, aux élèves et à la situation pédagogique, la conduite d'enseignement de l'enseignant et ses processus de pensée relatifs (Leuchter, 2009; Wanlin \& Crahay, soumis ; Wanlin, Lafontaine \& Crahay, soumis). Ainsi les connaissances systématiques se transformeraient progressivement, sous l'effet de l'expérience professionnelle, pour devenir mieux adaptées à l'action et à la réflexion pédagogique. Bref les croyances des enseignants nourrissent l'agir et la réflexion professionnels et s'en alimentent. Se constituant à partir des expériences vécues et des informations, savoirs, modèles de pensée reçus et transmis par la tradition, l'éducation et la communication sociale, les croyances ou représentations auraient une visée pratique d'organisation, de maîtrise de l'environnement et d'orientation des conduites. Participant à la construction d'une réalité commune à un ensemble social ou culturel donné, elles donneraient une vision fonctionnelle du monde qui permet à l'individu ou au groupe de donner un sens à ses conduites, de comprendre la réalité à travers son propre système de référence, donc de s'y adapter et de s'y définir une place. Dans cette perspective, il convient de considérer que les connaissances, conceptions, croyances ou représentations (11) peuvent remplir des fonctions plurielles et, plus particulièrement, selon l'analyse d'Abric (1994), des fonctions de savoir, d'identité, d'orientation et de justification. Nous reprenons cette analyse en l'adaptant.

La première fonction des croyances consiste à chercher à donner sens aux expériences vécues. Pour comprendre et expliquer les réalités auxquelles ils se 
confrontent dans leur vie quotidienne, les individus recourent à des idées d'origine et de statut divers. II y a donc un souci de compréhension, une tentative de donner sens aux actions accomplies et à celles à entreprendre. Ainsi, pour Kagan (1992), confrontés aux incertitudes et ambiguïtés inhérentes au monde scolaire et à la vie en classe (12), les enseignants s'efforcent de conférer de l'assurance à leur action en se donnant une pédagogie personnelle, plus ou moins cohérente. Cette quête de sens peut conduire à des acquisitions de connaissances, à l'élaboration de nouvelles croyances ou à l'ajustement de convictions anciennes et éventuellement au remodelage radical du système de croyances de l'individu. Nous parlerons de fonction épistémique des croyances, en référence à leur mobilisation dans le processus de compréhension mis en œuvre par les individus (et, en particulier, les enseignants), mais aussi en référence au rôle de matrice qu'elles sont supposées jouer dans la sélection et l'acquisition de connaissances nouvelles. Nombreux sont les auteurs qui attribuent cette fonction aux croyances des enseignants (Calderhead \& Robson, 1991 ; Clark \& Peterson, 1986 ; Grant, 2008 ; Grant \& Agosto, 2008 ; Howard \& Aleman, 2008 ; Kagan, 1992 ; Pajares, 1992 ; Borko \& Putnam, 1996 ; Richardson, 1996 ; Richardson \& Placier, 2001 ; Schommer, 1990 ; McDiarmid \& Clevenger-Bright, 2008 ; Woolfolk Hoy, Davis \& Pape, 2006). Autrement dit, les croyances offriraient aux individus un cadre de compréhension des événements, permettant d'intégrer des éléments nouveaux en cohérence avec leur fonctionnement cognitif et les valeurs auxquelles ils adhèrent. Sur le plan collectif, les croyances ou représentations définiraient un cadre de référence commun à un groupe social ou professionnel, permettant un partage d'idées et d'affects, ce qui favorise la communication à l'intérieur du groupe et en renforce la cohésion.

La deuxième fonction est identitaire: le partage de croyances communes permet aux individus ou groupes d'individus de se situer dans le champ social. En compatibilité avec des systèmes de normes et de valeurs socialement et historiquement déterminés, elles permettraient l'élaboration d'une certaine identité sociale et personnelle (Abric, 1994 ; Mugny \& Carugati, 1985). Les croyances ou représentations sont donc à concevoir comme des constructions cognitives permettant aux groupes sociaux de se donner une identité spécifique (13). En partageant les mêmes idées sur un objet social donné, un groupe social se constitue et se démarque, par ce fait même, d'un autre groupe. Dans certains cas, défendre une croyance (parfois envers et contre tout), c'est défendre son identité et son appartenance à un groupe social.

Les représentations ou croyances des enseignants remplissent une fonction que l'on peut qualifier de normative dans la mesure où il leur arrive de jouer un rôle d'orientation, de guide des comportements, des actions et des pratiques. Le plus souvent mise en avant dans les discours psychopédagogiques, cette fonction suppose que l'on attribue une valeur prescriptive aux croyances. Ceci est plausible en vertu de leur caractère socialement partagé : traduisant ce qu'il est légitime de penser sur un objet donné au sein de son groupe social d'appartenance, la croyance définirait ce qui est licite, tolérable ou inacceptable comme comportement ou pratique dans un contexte social donné. Par ailleurs, nous l'avons vu, les croyances opèrent - à l'instar de toute connaissance antérieure - en tant que schème cognitif, jouant un rôle actif dans la sélection et le filtrage des informations ainsi que dans l'interprétation des situations visant à rendre cette réalité conforme à la croyance.

Enfin la quatrième fonction possible des représentations ou des croyances est justificatrice ou autodéfensive (Abric, 1994). Ainsi certains comportements sont expliqués ou justifiés au regard des représentations qui ont présidé à leurs 
manifestations (Avigdor, 1953 ; Doise, 1973 ; Wilson \& Katayani, 1968). Comme on y reviendra plus loin, des recherches ont montré que, pour les jeunes enseignants, l'entrée en fonction est souvent source de difficultés, au point que la plupart d'entre eux connaissent un choc de la réalité (ou reality shock, voir Nault, 1999 ; Tardif \& Lessard, 1999 ; Veenman, 1984). Confrontés au décalage entre le métier rêvé ou imaginé et le métier réel (Nault, 1999), des enseignants développent un discours autodéfensif au sein duquel certaines croyances peuvent être mobilisées à des fins justificatrices.

\section{LES CROYANCES ET CONNAISSANCES DES ENSEIGNANTS PEUVENT-ELLES ÉVOLUER ?}

\section{Mise en perspective}

La question de la "malléabilité » des croyances et des connaissances des enseignants est cruciale dans la perspective de leur formation. On peut en effet considérer que les dispositifs de formation initiale, mais aussi les dispositifs de " recyclage » ou de formation continue des enseignants tablent sur un postulat de malléabilité. Plus fondamentalement, le présupposé fondamental de bon nombre de dispositifs de formation est le suivant: en transformant les croyances (et/ou conceptions) des enseignants, en améliorant leurs connaissances disciplinaires (subject matter knowledge and beliefs) et pédagogiques (à la fois leurs general pedagogical knowledge and beliefs et leurs pedagogical content knowledge and beliefs), on améliore leurs pratiques (14). Ce présupposé fondamental peut être décomposé en deux propositions :

- les croyances des enseignants déterminent leurs pratiques ;

- les croyances des enseignants sont modifiables ou, autrement dit, elles peuvent évoluer.

Dans le cadre de cette note de synthèse, nous nous intéressons principalement à l'examen des recherches portant sur la seconde proposition, laissant à plus tard (comme déjà indiqué ci-dessus) l'examen systématique des relations (complexes) entre croyances et pratiques. II nous faudra toutefois aborder la question importante de la priorité du changement des croyances par rapport aux pratiques.

Que les croyances et connaissances des enseignants soient modifiables est une affirmation fort controversée dans la littérature de recherche en éducation. Un premier examen des études empiriques donne l'impression qu'il y a autant de résultats établissant une réponse négative que de données autorisant une position optimiste. Dans un article de 1998 intitulé "How teachers change ", Richardson remarque que l'affirmation "Teachers don't change, they resist change " cohabite, dans la littérature, avec l'affirmation contradictoire "Teachers change all the time ". Plus précisément, elle note que des auteurs comme Duffy et Roehler (1986) ou Fullan (1991) affirment, sur la base de leurs recherches, que les enseignants sont résistants au changement. Le travail historique de Cuban (1984 ; voir aussi Cuban, 1988) a beaucoup contribué à l'idée d'une persistance quasi immuable des formes d'enseignement; il en parle comme d'une continuité obstinée de la forme d'instruction traditionnelle, résistante aux multiples tentatives de réforme pédagogique s'efforçant d'introduire le plan Dalton, la pédagogie du projet, le travail d'équipe, la classe ouverte, etc. Cette analyse a été reprise par Gage (1986) qui en tire argument pour conclure au " caractère inexpugnable de cette forteresse qu'est l'enseignement traditionnel » (p. 420). À l'inverse, dans un article intitulé "Significant and worthwhile change in teaching practice», Richardson (1990) 
observe, chez divers enseignants, des modifications dans leurs façons d'enseigner, allant de changements mineurs à des transformations radicales ; un constat qu'elle réitère dans une étude ultérieure (Richardson, 1994).

Deux clarifications conceptuelles s'imposent dès à présent. II convient de distinguer, d'une part, changement de croyances et changement de pratiques et, d'autre part, modifications de surface (first order change dans la littérature anglosaxonne) et transformations en profondeur (second order change ou structural changes (15)). Dans une synthèse intitulée "Research on teacher education " et publiée dans la troisième édition du Handbook of research on teaching, Lanier et Little (1986) concluent que changer le comportement et les pensées des enseignants est possible. II importe cependant de situer cette conclusion dans son contexte historique. On se trouve, au moment de la publication de ce Handbook, à l'apogée des recherches processus-produit et la revue de synthèse de Lanier et Little porte principalement sur des modifications de procédures d'enseignement, donc des modifications de premier ordre ou des changements de surface. Or, pour Richardson et Placier (2001), personne ne doute de la capacité des enseignants à modifier l'une ou l'autre stratégie comportementale spécifique (cf. p. 915). Pour notre part, cette prise de position nous paraît sous-estimer la difficulté des changements comportementaux, une question que l'un de nous a traitée jadis dans une note de synthèse (16) intitulée "Contraintes de situation et interactions maître/élèves: changer sa façon d'enseigner, est-ce possible ? » (Crahay, 1989). Toutefois nous nous accordons avec Richardson et Placier pour considérer que la question du changement des croyances se pose désormais dans un contexte paradigmatique différent de celui marqué par le comportementalisme des années soixante-dix et quatre-vingt.

Shulman (1987) et Fenstermacher $(1979,1986)$ ont été deux des principaux artisans de ce bouleversement qui s'inscrit dans le passage de l'ère béhavioriste à la période encore actuelle, dominée par le cognitivisme. De façon précise, il convient de faire remonter ce changement paradigmatique à 1974, date à laquelle le panel 6 du National institute of education (NIE), présidé par Shulman, sort un rapport intitulé Teaching as clinical information processing. Ce rapport insiste sur la nécessité de mener des recherches dont le but est de comprendre la vie mentale des enseignants, de saisir les processus cognitifs qu'ils mobilisent pour faire face à un environnement complexe que Doyle (1975, 1977, 1979 et 1986) caractérise par la multidimensionnalité, la simultanéité, l'immédiateté et l'imprévisibilité. L'influence de ce panel 6 sur la recherche sur l'enseignement fut considérable. On lui attribue le déclin des recherches processus-produit et l'émergence du courant baptisé teacher thinking, un courant dont Shulman (1987) critiquera les lacunes en soulignant que les recherches menées dans cette perspective entre 1975 et 1985 (pour une synthèse de ce courant, voir Clark \& Peterson, 1986 ou Borko \& Shavelson, 1990) ignorent le contenu enseigné. Comme indiqué ci-dessus, pour Shulman, la compréhension du processus d'enseignement ne peut faire l'impasse des diverses sortes de connaissances activées par les enseignants pour conduire la classe et tenter de susciter l'apprentissage des élèves.

À tous ces bouleversements théoriques, s'ajoute l'influence de la réflexion philosophique de Fenstermacher (1979, 1983, 1984a, 1984b, 1985, 1986). Celle-ci porte principalement sur l'usage que l'on peut faire des recherches empiriques pour penser la formation des enseignants. Anticipant les critiques adressées aujourd'hui à l'éducation basée sur la preuve (Saussez \& Lessard, 2009), ce penseur de l'éducation s'insurge contre un applicationnisme strict qui, de facto, contraint les enseignants à se conformer aux recommandations tirées des résultats de recherches et les transforme en exécutant, voire en "petit soldat " obligé 
d'obéir aux standards de qualité définis en termes de best practices. Pour Fenstermacher, l'enseignant doit être un professionnel qui sait pourquoi il agit de telle façon plutôt que de telle autre. Autrement dit, la qualité, le bien-fondé et la cohérence des arguments et/ou des justifications qui sous-tendent le choix d'une pratique sont tout aussi importants que les effets supposés de cette pratique. Le titre de son article de 1985 est à cet égard instructif : "Who decides the worth of educational research? ", une question qu'il complète par une autre répétée dans la plupart de ses textes : les résultats des recherches sur l'enseignement peuvent-ils dicter à eux seuls les programmes de formation des enseignants et ce qu'il convient de considérer comme le bon enseignement ? Cette réflexion séminale est sous-jacente à tous les travaux empiriques qui s'attachent à appréhender dans quelle mesure les croyances et les connaissances des enseignants sont modifiées et/ou se transforment en profondeur.

C'est le mérite de Richardson et Placier (2001) d'avoir placé leur revue des recherches sur les croyances et connaissances des enseignants dans ce contexte paradigmatique. À nos yeux, cette revue de synthèse fait encore autorité, presque dix ans après, quels que soient les apports des synthèses ultérieures (Evertson \& Weinstein, 2006 ; Munby, Russell \& Martin, 2001 ; Woolfolk Hoy, Davis \& Pape, 2006). Autre mérite de cette revue: les auteures catégorisent la recension des recherches en deux catégories principales, individual and small group change, d'une part, et staff development d'autre part, lesquelles se déclinent en sous-catégories (trois pour le premier groupe et deux pour le second). Pour notre part, nous proposons une structure en trois catégories:

- les recherches portant sur les changements naturels, qualifiés également de volontaires (voluntary and naturalistic changes (17)), un ensemble auquel nous adjoignons celles portant sur les stades de développement ponctuant - c'est le postulat - la carrière des enseignants ;

- les études s'attachant à mesurer les effets des programmes de formation des enseignants, dont la plupart concernent la formation initiale ;

- les recherches relevant du courant nommé staff development (18).

Nous introduirons, en outre, une section portant sur les croyances des enseignants débutants, juste après celle traitant des stades de développement; les recherches menées à ce sujet permettent de mieux comprendre les études évaluant les effets des programmes de formation.

\section{Qu'ils le veuillent ou non, les enseignants changent en cours de carrière}

C'est la conclusion principale qu'il est possible de tirer des travaux qui se basent sur les récits de vie des enseignants (études biographiques) ainsi que des nombreuses études qui ont tenté d'identifier des stades de développement au sein de la carrière des enseignants.

Parmi les études biographiques (pour une recension plus complète, voir Richardson \& Placier, 2001), on mentionnera celle de Butt, Raymond, McCue et Yamagisi (1992), celle de Bullough et Baughman (1997), ainsi que celle de Russell (1995). Dans la première, les chercheurs présentent deux études de cas. Les deux enseignants ont notamment été invités et ont accepté de fournir une description de leur contexte de travail ainsi que de leurs pratiques pédagogiques (notamment de leur façon de mettre en œuvre le curriculum), ainsi que de leur réflexion rétrospective sur leur vie personnelle et professionnelle. En ce qui concerne cette réflexion, il leur était demandé de mettre en relation leurs expériences passées avec leur vécu actuel. Butt, Raymond, McCue et Yamagisi 
(1992) mettent en évidence une évolution des deux enseignants, celle-ci étant influencée selon leurs dires par une diversité d'événements liés aux élèves, aux collègues, aux parents et même à leur vie personnelle. Bullough et Baughman (1997) tirent la même conclusion de leur étude collaborative de 8 ans, débutant au moment où Kerrie (Baughman) entre en fonction. Pour ces deux auteurs, il est certain que des changements se sont opérés dans la tête de Kerrie, dans ses croyances et dans son identité professionnelle (appréhendés à travers l'analyse des métaphores qu'elle utilise pour se définir et pour décrire sa pratique) et que ceux-ci sont indissociables de son évolution personnelle. Autrement dit, développements personnel et professionnel seraient indissociables. Quant à Russell (1995), formateur d'enseignants, il est retourné enseigner la physique pendant deux ans dans une high school et s'est donc engagé dans une self-study de laquelle il conclut à l'importance de l'expérience pratique, eu égard au développement professionnel. Cette idée est chère à Russell qui la partage avec son collègue Munby (cf. en particulier Munby \& Russell, 1994, texte dans lequel ces auteurs développent le concept d'authority of experience) ; elle est à nouveau exprimée dans un article de 1998 (Munby, Cunningham \& Chin, 1998).

Que dire de ces recherches ? Le constat que les enseignants évoluent sur le plan professionnel ne nous paraît guère contestable. Les recherches contrastant les experts et les novices ou les enseignants chevronnés et les enseignants débutants (cf. notamment l'étude de Russell, Munby, Spafford et al., 1988) le montrent abondamment. II y a donc là une sorte d'évidence. Quels sont les facteurs contribuant à cette évolution ? Là est bien la question importante. Les études de Russell (1988), Bullough et Baughman (1997) nous paraissent tellement exceptionnelles qu'il nous semble difficile de pouvoir généraliser les constats qui y sont opérés. À titre d'argument, on retiendra que, dans la préface du livre de Bullough et Baughman (1997), Berliner souligne que Kerrie est le type d'enseignante dont tous les parents rêvent pour leurs enfants. Quant à Russel (1988), il décide de reprendre du service pour mettre à l'épreuve de la réalité ses conceptions de formateur d'enseignant; la démarche est admirable, ce qui ne lui confère pas ipso facto un certificat de validité scientifique. Notons, par ailleurs, que d'autres chercheurs observent une permanence dans le temps de la façon d'enseigner de divers individus ; Richardson et Placier (2001) citent à ce propos l'étude de cas de Louden (1991) ainsi que le travail de Zahorik (1990) rassemblant quatre études de cas. Au final, il semble que l'on puisse conclure à la fois à un mélange de stabilité et de variabilité dans le développement professionnel des enseignants. Quelles facettes restent constantes et lesquelles évoluent? Dans l'état actuel des recherches, il n'est pas possible de répondre à cette question tant l'idiosyncrasie des enseignants semble importante. Cette caractéristique est soulignée par plusieurs chercheurs. Richardson et Placier (2001) citent à ce propos les études de Ball et Goodson (1985), de Munby et Russell (1992). II faut faire une mention spécifique à l'étude d'Ashton (1984) qui observe que les enseignants caractérisés par une haute perception de leurs compétences (high self-efficacy) sont davantage enclins à changer leurs pratiques que les autres. Enfin, à nouveau selon Richardson et Placier (2001), des chercheurs ont proposé des typologies d'enseignants en fonction de leur engagement dans la profession (Marks \& Gersten, 1998) ou en fonction de leur attitude face à l'introduction d'un nouveau curriculum (Lindblad, 1990). On trouve également des catégorisations analogues dans les recherches francophones (Crahay \& Donnay, 2001).

L'idée d'une évolution naturelle (et quasi inévitable) des enseignants en cours de carrière se retrouve dans les recherches s'attachant à identifier des stades de développement ponctuant cette évolution. Ici, à l'opposé des études précédemment citées, on postule que tous les enseignants évoluent en traversant les mêmes 
étapes. C'est clairement le cas de l'étude classique de Fuller (1969), reprise et prolongée par son travail avec Bown (Fuller \& Bown, 1975) qui, pour rappel, distingue quatre stades: un stade de la formation (preteaching) caractérisé par beaucoup d'idéalisme, un stade de la survie correspondant à l'entrée en fonction et marqué par un choc de la réalité dont les effets sont dévastateurs par rapport aux idéaux initiaux, un stade ciblé sur les pratiques et situations d'enseignement et, enfin, un stade terminal où les enseignants manifestent des préoccupations centrées sur les élèves, leurs apprentissages et leur épanouissement (19). Plus récemment, Nias (1989) a retrouvé, dans une étude menée auprès de 99 enseignants, à peu près les mêmes étapes que Fuller. Dans la même veine, on peut encore ranger deux études. À partir d'une étude menée sur 50 enseignants, Mevarech (1995) distingue cinq stades dans le développement professionnel allant d'une étape de survie à une étape d'implémentation réfléchie du curriculum, cette dernière étape se couplant avec des manifestations de changements conceptuels. Feiman-Nemser (1983), quant à elle, identifie quatre stades (pretraining, preservice, induction et inservice). Mentionnons encore le travail de Berliner (1994) qui distingue cinq niveaux d'expertise (novice, débutant avancé, compétent, compétent avancé - ou proficient - et enfin expert).

Synthétisant les études sur la socialisation professionnelle des enseignants, Nault (1999) propose un modèle en cinq phases. II y aurait d'abord la phase du métier rêvé ou imaginé, phase qui précède l'entrée en formation initiale et au cours de laquelle l'adolescent se construit une image de ce qu'il veut devenir et se forge un projet professionnel. Au cours de la seconde phase, celle de la formation initiale, le futur enseignant acquiert les savoirs, savoir-faire et savoir-être de sa future profession. Parallèlement, il subit l'influence de forces externes qui visent à modeler son "moi professionnel » à l'aune des théories pédagogiques. Ensuite viendrait la phase de l'insertion professionnelle. D'une importance capitale pour le devenir des enseignants, elle peut être décomposée en trois étapes: primo l'étape d'euphorie anticipatrice, suivie secundo de l'étape du " choc de la réalité " et enfin, tertio, l'étape de la consolidation des acquis pendant laquelle, au fur et à mesure des succès, ce nouvel enseignant se construit des structures permanentes de fonctionnement et de réflexion. La quatrième phase correspondrait à une socialisation plus personnalisée. Au sein de sa classe, l'enseignant continue à se développer professionnellement en fonction des expériences, de ses essais et erreurs. Enfin l'ultime phase est appelée phase de socialisation rayonnante car, après avoir surmonté avec succès des situations complexes, l'enseignant est amené à partager son expertise avec ses collègues. II est au sommet de son art.

Dans toutes ces recherches, évolution est synonyme de développement et, partant, de progrès, voire d'épanouissement professionnel. Ceci est particulièrement évident dans la synthèse que propose Nault (1999) de cette littérature. En lisant la succession des phases, on ne peut s'empêcher de penser qu'il s'agit plus là de la projection d'un idéal que de la description d'étapes de développement. Au terme d'une évolution parfois longue et semée d'embûches qu'il surmonte, l'enseignant atteindrait un point oméga, celui de l'expertise. De plus, poussé par un idéal de solidarité, il rayonne autour de lui en dispensant son savoir professionnel vers ses collègues. On peut évidemment rêver qu'il en soit ainsi, mais il faut craindre que tous les enseignants n'atteignent pas ce stade ultime d'épanouissement. Les travaux sur la dépression et le burn out des enseignants sont là pour nous ramener au réalisme. Seule exception à cette tendance à édulcorer la réalité, l'étude de type rétrospectif menée par Huberman, Grownver et Martin (1989) sur 160 enseignants suisses de l'enseignement secondaire invités à retracer au cours d'entretiens semi-structurés leur parcours professionnel : le dernier stade est celui 
du désengagement. De surcroît, Huberman et ses collaborateurs soulignent que tous les enseignants ne passent pas par les six stades qu'il a pu identifier, considérant que l'évolution des enseignants s'inscrit dans un contexte et dans une histoire de vie professionnelle marqués par l'émergence et parfois le retrait de diverses réformes, ces événements affectant nécessairement leur devenir.

Sans doute faut-il retenir de ces recherches que les enseignants évoluent. En revanche, il est peu probable que tous traversent les mêmes étapes de développement, comme il est douteux que toutes les carrières d'enseignants s'achèvent en apothéose. Le modèle piagétien de développement linéaire est désormais abandonné en psychologie du développement et remplacé par une conception en vagues qui se chevauchent. Selon cette métaphore due à Siegler (1999), à chaque phase de développement, dans chaque sous-domaine, l'enfant dispose de plusieurs stratégies cognitives, qui, à l'image des vagues s'approchant par va-et-vient progressifs du rivage, sont susceptibles de se succéder rapidement, mais aussi de se chevaucher et donc d'entrer en compétition. Avec l'expérience et selon les situations, l'enfant apprend à choisir l'une ou l'autre façon de procéder. On peut supposer que ce modèle général, mis en évidence par l'observation des enfants, est valable - au moins, partiellement (20) - pour les enseignants. C'est ce que laissent penser les études qui se sont efforcées d'identifier des étapes dans le développement des enseignants confrontés à des innovations curriculaires. Ainsi, dans une étude de Schifter (1995), pour s'approprier un curriculum constructiviste de mathématiques, des enseignants traversent quatre étapes au cours desquelles leurs conceptions de l'enseignement des mathématiques se transforment. Ils passent d'une conception ramenant cette discipline à une accumulation de faits à une conception où l'investigation systématique joue un rôle central. De même, Hollingsworth (1989), observant comment des étudiants en formation s'approprient l'enseignement de la lecture, constate que ceux-ci doivent maîtriser les techniques de conduite de la classe avant de pouvoir travailler leurs croyances pédagogiques et, tout particulièrement, les croyances pédagogiques portant sur le contenu. Bref, avec ces recherches, nous avons la preuve que, dans certaines circonstances, les croyances des enseignants peuvent évoluer.

On retiendra encore des études portant sur les stades de développement la notion de choc de la réalité (cf., pour une synthèse, Veenman, 1984, mais aussi Tardif \& Lessard, 1999), signifiant que, pour la plupart des enseignants débutants, l'insertion professionnelle s'apparente à un désenchantement brutal au cours duquel il leur faut renoncer aux "belles idées » et croyances qu'ils ont au terme de leur formation initiale, mais aussi - semble-t-il - avant même d'entamer celle-ci. C'est, en effet, ce que tendent à indiquer les études menées sur les croyances des futurs enseignants à l'entrée de la formation, études que nous passons en revue dans la section suivante.

\section{Les conceptions des futurs enseignants à l'entrée de la formation}

Selon plusieurs recherches (par exemple, Book, Byers \& Freeman, 1983 ; Weinstein, 1988, 1989 ; Pines \& West, 1986), les futurs enseignants manifestent dès l'entrée en formation une grande confiance dans leur capacité à enseigner. Selon Richardson (1996), ce résultat serait flagrant, à la fois à travers les nations et à travers le temps, c'est-à-dire à partir des années cinquante jusqu'au milieu des années quatre-vingt-dix. Weinstein (1988) parle à ce sujet d'un "optimisme irréaliste ". Les étudiants sous-estimeraient largement la complexité du métier. Jensen (1998) se demande dans quelle mesure cet optimisme est susceptible de les amener à un investissement minimal dans la formation. 
Plus récemment, Montgomery, Legault, Gauthier et Bujold (1999) ont évalué le sentiment d'efficacité personnelle de 431 étudiants canadiens via un questionnaire administré durant la toute première semaine de formation. Une partie des étudiants étaient inscrits au baccalauréat en enseignement secondaire, l'autre en enseignement primaire. Leurs résultats vont dans le sens de ceux des études précédentes, mais apparaissent plus nuancés : 94,2 \% des participants estiment globalement être plus compétents qu'un enseignant débutant. II apparaît cependant que les scores relatifs aux compétences liées aux relations avec les élèves sont beaucoup plus élevés que ceux correspondant aux compétences requérant un savoir-faire spécifique (l'évaluation des apprentissages, la gestion de la classe, la planification des activités, l'intervention envers les élèves plus « difficiles » et l'analyse des pratiques pédagogiques). Les auteurs de l'étude estiment qu'un tel sentiment global de confiance "peut être le gage d'attitudes positives et de stratégies proactives efficaces lorsque surgissent les problèmes inhérents à la tâche » (Montgomery, Legault, Gauthier et al., 1999, p. 161) mais que, poussé à l'extrême, " ce sentiment peut s'apparenter à une pensée magique, soit la conviction que les problèmes qui affligent les autres nous épargneront » (p. 161).

D'autres études suggèrent qu'en début de formation, les étudiants conçoivent l'enseignement-apprentissage comme un processus passif ou transmissif. Pour eux, enseigner consiste à transmettre des faits et l'apprentissage procède par la mémorisation de ces mêmes faits (Black \& Ammon, 1992 ; Brookhart \& Freeman, 1992 ; Calderhead, 1988 ; Holt-Reynolds, 1992 ; Mahlios \& Maxson, 1998 ; McDiarmid, 1990 ; Russel, 1988). À ce sujet, Strauss et ses collègues (Strauss, 1993 ; Strauss \& Shilony, 1994), résument les conceptions des élèves maîtres en termes de modèles mentaux combinant croyances épistémiques et conceptions pédagogiques de type transmissif. Ils écrivent:

« Un postulat de base de leurs modèles mentaux suppose que la connaissance existe à l'extérieur de l'esprit des enfants. Elle se situe dans l'esprit du professeur, dans les livres, etc. En général, les enfants ont des connaissances, mais elles sont souvent incomplètes ou incorrectes. Parfois, ils n'ont pas de connaissances au sujet du domaine qu'on est en train de leur enseigner. Cette position mène à considérer l'enseignement (et l'apprentissage des enfants qui en découle) comme étant constitué de deux parties principales. Premièrement, le professeur doit trouver un moyen de faire entrer la connaissance dans l'esprit des enfants. Deuxièmement, une fois que cette connaissance entre dans leur esprit, l'enseignant doit agir de façon telle que les enfants vont déplacer la nouvelle matière de l'endroit où elle est entrée et l'emmener à l'endroit où elle va être stockée, ajoutant ainsi celle-ci au stock actuel de concepts, compétences, etc. déjà appris »(Strauss, 1993, p. 154).

Relevons encore des études relatives aux conceptions du "bon enseignant » ou du «bon enseignement » qui suggèrent une tendance à minimiser les aspects académiques ou cognitifs et à privilégier les aspects interpersonnels ou affectifs de l'enseignement (par exemple, Brookhart \& Freeman, 1992 ; Montgomery, Legault, Gauthier et al., 1999). Ainsi, dans une étude menée par questionnaire auprès de 473 candidats à l'enseignement (dont $78 \%$ de femmes), Book, Byers et Freeman (1983) constatent que, pour une majorité d'entre eux, l'enseignement est perçu comme une forme de parentalité ayant pour but de développer le concept de soi des élèves. Clairement, à travers leurs réponses, les futurs enseignants sous-estiment la facette " académique " du métier ainsi que les problèmes liés à la gestion du groupe, privilégiant les aspects psychologiques et la socialisation des élèves. Ils considèrent aussi qu'enseigner ne s'apprend pas ; pour progresser dans le métier, il faut se nourrir de ses intuitions et de ses propres expériences. En ce qui concerne les attentes à l'égard de la préparation et de la formation, la 
majorité des étudiants accorde une importance primordiale à l'apprentissage sur le tas et aux stages professionnels. Ils les considèrent comme les deux principales sources de connaissances professionnelles. D'autres sources du même type sont désignées dans l'ordre d'importance décroissante suivant: les cours de psychologie de l'éducation, son expérience personnelle en tant qu'élève, les lectures personnelles et les cours sur les fondements socio-philosophiques de l'éducation. Enfin, confirmant les autres études du même type, une grande part des étudiants s'estime capable d'enseigner et exprime ne pas ressentir le besoin d'avoir des connaissances professionnelles pour le faire. Ce sentiment élevé de confiance en soi se retrouve sans distinction de genre et de niveau d'enseignement (préscolaire, primaire ou secondaire) auquel les étudiants se destinent.

Les tendances qui se dégagent des études sur les croyances relatives à l'enseignement et à l'apprentissage sont à considérer avec nuance. Les futurs enseignants sont loin de former un groupe homogène. Plusieurs recherches concluent à la variété des conceptions des futurs enseignants (par exemple, Gurney, 1995 ; Schmidt \& Kennedy, 1990). Ainsi, explorant les métaphores de 124 étudiants inscrits en baccalauréat en enseignement primaire, Leavy, McSorley et Boté (2007) les ont classées principalement en termes de conceptions béhavioristes empiristes, cognitivistes constructivistes et situées (perspective socio-historique). À l'entrée en formation, ils relèvent les proportions respectives suivantes concernant la répartition de ces conceptions : $49 \%, 24 \%$ et $18 \%$. Par ailleurs, bien qu'il existe des points communs entre les futurs enseignants du secondaire et du primaire, plusieurs études (citées par Jensen, 1998) montrent des différences. Par exemple, les étudiants qui se préparent à l'enseignement primaire auraient une conception de l'enseignement davantage centrée sur l'élève, tandis que ceux qui enseigneront dans le secondaire accorderaient plus d'importance à la matière (Book \& Freeman, 1986).

\section{Les croyances des enseignants changent-elles en cours de formation ?}

Étude de l'effet des curriculums de formation

sur l'évolution des croyances et des connaissances des enseignants

Richardson (1996) d'abord, Borko et Putnam (1996) ensuite et Richardson et Placier (2001) enfin ont fait la recension des recherches qui se sont données pour objet d'établir l'effet des curriculums de formation initiale sur l'évolution des croyances des futurs enseignants. Leurs conclusions sont pessimistes, au point que Borko et Putnam (1996) consacrent une part importante de la discussion des résultats aux obstacles (impediments) aux changements. Richardson et Placier (2001) sont, quant à eux, plus modérées en parlant de la difficulté à faire évoluer les croyances des jeunes enseignants. Pour notre part, tout en rejoignant la conclusion de Richardson et Placier (2001), il nous semble surtout que la diversité des résultats et des méthodes de recherche force à la prudence.

Étayons cette position. On peut d'abord noter que, si des chercheurs concluent que des changements se sont opérés en cours de formation (par exemple, Hollingsworth, 1989 ; Ammon, 1991 ; Levin \& Ammon, 1992 ; Winitzky, 1992 ; Jones \& Vesilind, 1996; Winitzky \& Kaufchak, 1997), une majorité d'études concluent à la stabilité des croyances (par exemple, Zeichner, Tabachnick \& Densmore, 1987 ; Feinman-Nemser, 1990 ; Tickle, 1991 ; Wilcox, Shram, Lappan et al., 1991 ; McDiarmid, 1992 ; Munro, 1993 ; Olson, 1993 ; Tilema \& Knol, 1997 ; Crahay \& Ory, 2006 ; Saban, Kocbeker \& Saban, 2007). À titre d'illustration de cette catégorie de recherches à la conclusion négative, on mentionnera tout particulièrement le programme de recherche TELT (21), dans le cadre duquel un 
nombre conséquent d'études de cas d'enseignants en formation ont été réalisées dans le but d'appréhender l'évolution de leurs croyances envers l'hétérogénéité des élèves et les effets de ces changements de croyances sur le processus d'enseignement-apprentissage. Les chercheurs n'ont noté aucun changement substantiel dans ces croyances au cours de la formation.

Plus nuancés, d'autres chercheurs tirent des conclusions signalant que certains des étudiants évoluent dans leurs croyances (22) (Bolin, 1990 ; Korthagen, 1988 ; Markes, 1995), tandis que d'autres signalent des frémissements d'évolution après une phase de "résistance " (Civil, 1996; Harington \& Hathaway, 1994). Enfin il faut encore signaler les études qui, mesurant différentes catégories de croyances, observent des changements pour certaines d'entre elles et non pour d'autres (Korthagen, 1988 ; Feiman-Nemser, McDiarmid, Melnick et al., 1989 ; Woolfolk Hoy \& Burke Spero, 2005). II faut regretter qu'une majorité d'études se soient concentrées sur l'évolution d'un seul type de changement, car on peut faire l'hypothèse - nous y reviendrons - que certaines croyances sont plus difficiles à modifier que d'autres. Or, si plusieurs études portent, eu égard aux idées pédagogiques en vigueur au cours des dernières décennies, sur le développement chez les étudiants de conceptions constructivistes de l'apprentissage et/ou de l'enseignement, d'autres portent sur le développement d'une pensée réflexive, et d'autres encore sur les croyances concernant l'enseignement des mathématiques, le redoublement ou l'éducation multiculturelle. Si l'on croise cette deuxième dimension avec la précédente concernant la tendance des résultats, on peut dresser le tableau situé en annexe 1.

Soulignons également que l'ampleur des études est extrêmement variable. Ainsi Olson (1993) tire la conclusion que les futurs enseignants ne changent pas leurs croyances à propos de "la bonne façon d'enseigner " suite aux cours donnés en formation, en se basant sur l'étude de cas de deux étudiants. À l'opposé, l'étude menée en Belgique par Crahay et Ory (2006), concernant les croyances relatives au redoublement, porte sur 650 élèves maîtres. De même, les méthodes utilisées pour appréhender les croyances fluctuent également: questionnaire (Crahay \& Ory, 2006) ; entretien (par exemple, Korthagen, 1988) ; métaphores, cartes conceptuelles (par exemple, Jones \& Vesilind, 1996 ; Winitzky, 1992 ; Winitzky \& Kaufchak, 1997), dilemmes ou situations problématiques à résoudre (par exemple, Korthagen, 1988 ; Zeichner, Tabachnick \& Densmore, 1987), etc. Notons que la conclusion, à savoir s'il y a eu changement ou pas, est toujours affaire d'appréciation. Nous en fournirons deux exemples. Saban, Kocbeker et Saban (2007), qui ont analysé les métaphores relatives au concept " enseignant » exprimées par 1142 futurs enseignants, concluent que l'hypothèse selon laquelle les étudiants de dernière année choisiraient plus d'images métaphoriques centrées sur l'élève n'est pas confirmée. Toutefois des changements dans les réponses des étudiants ont été constatés. Parmi les six catégories métaphoriques dominantes identifiées dans cet échantillon (l'enseignant en tant que transmetteur de savoir, sculpteur/artisan, cultivateur/artisan, conseiller, "facilitateur » et leader coopératif), deux d'entre elles différencient les étudiants entrants et les étudiants sortants: les premiers expriment plus d'images orientées vers le développement (cultivateur/artisan) tandis que les derniers proposent davantage de métaphores illustrant la facilitation.

Dans le contexte belge, Crahay et Ory (2006) ont réalisé une étude quantitative auprès de futurs enseignants du primaire de six HEP (Hautes écoles pédagogiques) afin d'évaluer dans quelle mesure leurs croyances à propos du redoublement changent au cours de la formation. Les informations recueillies montrent que les étudiants de première année se déclarent clairement en faveur du redoublement, 
tout particulièrement en tout début de formation, alors que l'avis de leurs condisciples de deuxième et de dernière année est en moyenne plus mitigé. Cependant ces chercheurs observent que l'évolution de ce positionnement général concernant le redoublement ne repose pas sur un développement des conceptions quant aux effets spécifiques (scolaires, cognitifs, affectifs et sociaux) du redoublement, ni sur une transformation de leurs croyances quant aux causes des échecs scolaires. Notamment, en fin de formation comme en début, les futurs enseignants incriminent les élèves (leur manque d'effort, leur maturité ou encore leurs faibles aptitudes) ainsi que leurs familles et rechignent à mettre en cause l'enseignement reçu ou même le fonctionnement général de l'école. De plus, ils persistent à nier le poids de l'influence de l'origine socioculturelle des élèves dans la fabrication des échecs scolaires. In fine Crahay et Ory (2006) concluent qu'il n'y a pas eu de transformation en profondeur des croyances de la plupart des enseignants en formation.

Évidemment, les auteurs ne se contentent pas d'enregistrer l'échec des tentatives de transformation des croyances des enseignants. Chacun, à sa manière, tente une explication. Nous reviendrons sur ce point, crucial, dans la discussion.

Études relatives à l'effet des cours

sur les croyances des enseignants en formation

Un certain nombre d'études porte sur l'effet spécifique de certains cours sur les croyances et connaissances des enseignants en formation. Les conclusions sont, là également, plutôt pessimistes. Ainsi McDiarmid (1992) a observé que les croyances des étudiants par rapport à l'éducation multiculturelle changent peu suite à une série d'exposés sur le sujet. Holt-Reynolds (1992) a observé que les étudiants d'un cours de méthodologie de l'enseignement de la lecture rejetaient les idées du formateur centrées sur l'élève et le processus, et ce sur la base de leurs croyances préexistantes quant au "bon enseignement ", aux "bons sujets de leçon ", etc. L'auteur conclut à la primauté des croyances personnelles des étudiants sur les nouvelles méthodologies ou façons de penser prônées par les formateurs. D'autres recherches montrent que, là où on voulait les modifier, les croyances initiales se voient renforcées (voir par exemple Feiman-Nemser \& Buchmann, 1989).

Une série d'études ont mesuré l'impact de cours spécifiquement conçus pour changer les croyances des futurs enseignants. Dans ces études, les chercheurs étaient bien souvent eux-mêmes formateurs. Parmi celles-ci, on mentionnera celles de Ball (1989), Civil (1993) ainsi que celle de Simon et Mazza (1993) qui, toutes trois, montrent que les croyances d'un certain nombre d'étudiants ne se modifient pas dans la direction souhaitée. Ces auteurs questionnent la possibilité de changer les croyances en un cours ou même en un programme. Les résultats de Feiman-Nemser et Buchmann (1989) sont, eux, plus encourageants. Ces chercheurs ont évalué le changement des croyances de 91 étudiants suite à un cours donné en début de formation et concluent que celui-ci a eu un impact à différents niveaux. Ainsi, à la fin du cours, les étudiants considèrent que l'enseignement est plus complexe que ce qu'ils pensaient au départ et le lien entre l'enseignement et l'apprentissage commence à être perçu par eux. De même, les résultats des recherches menées par McDiarmid (1990), Ball (1988) et Comeaux (1992) montrent que lorsque les cours de pédagogie essaient explicitement de mettre en question les croyances préalables des futurs enseignants, des changements peuvent s'opérer et s'opèrent d'ailleurs effectivement. McDiarmid (1990) note toutefois que les croyances des enseignants s'avèrent extrêmement difficiles à changer ; en conséquence, il reste sceptique quant à l'impact que de tels cours peuvent avoir, en particulier en ce qui concerne leur transfert dans les pratiques des étudiants. 
Comme déjà indiqué ci-dessus, en formation, la dimension pratique est importante aux yeux des étudiants. Ceux-ci attendent davantage de leurs stages que des cours, estimant que c'est principalement, voire exclusivement, l'expérience de terrain qui est formatrice (cf. notamment Book, Byers \& Freeman, 1983 ; Crahay \& Ory, 2006 (23) ; Richardson-Koehler, 1988, cités par Richardson, 1996). Quant à savoir si le temps passé sur le terrain contribue à la transformation des croyances des élèves maîtres, le bilan des études est loin d'être encourageant. Les études de Hoy (1967) et de Hoy et Woolfolk (1990) montrent ainsi que les étudiants débutent la formation avec des croyances relatives à leur rôle d'enseignant orientées vers le contrôle (importance de maintenir la discipline et de contrôler les élèves). Ces dernières changent - du moins en apparence - au cours de la formation, évoluant vers une position plus humaniste. Cependant, lorsque les futurs enseignants vont en stage, ils "renversent " leurs croyances vers une position plus rigide. Les auteurs émettent l'hypothèse que ce changement est dû à l'existence d'une idéologie du contrôle, présente dans les écoles, qui affecte les étudiants à travers le processus de socialisation.

Sur la base de ces travaux, Cochran-Smith (1991) suggère que les stagiaires sont marqués par leurs années passées à l'école en tant qu'élèves, une idée développée antérieurement par Lortie (1975). Bien qu'ils expriment des avis humanistes durant les cours de formation, leurs croyances préexistantes, issues essentiellement de leur expérience scolaire, prévalent lorsqu'ils renouent avec la réalité des classes durant leurs stages. Ainsi, en définitive, les expériences faites dans le contexte scolaire renforceraient les croyances que les enseignants en formation ont acquises du fait d'avoir été élèves eux-mêmes. Notons que, dans le cadre de ces études, on est en droit de s'interroger sur la profondeur des transformations des croyances générées pendant la formation théorique.

Leavy, McSorley et Boté (2007) ont étudié l'évolution au cours de la première année de formation des croyances de deux cohortes d'étudiants à propos de l'enseignement et de l'apprentissage, et ceci à travers l'expression de leurs métaphores sur le sujet. Les étudiants de la première cohorte ont bénéficié de cours de méthodologie, de séances de micro-enseignement ainsi que d'activités réflexives au cours du premier semestre. Ils ont ensuite réalisé des stages à raison d'un jour par semaine, ceux-ci alternant avec des moments de réflexion sur la pratique, au cours du second semestre. Les seconds ont également bénéficié de cours de méthodologie, mais ne sont pas allés sur le terrain. Les résultats de cette étude montrent notamment qu'en fin d'expérience, les métaphores béhavioristes prévalant en début d'année demeurent dominantes parmi les étudiants de la première cohorte. À l'inverse, chez les étudiants de la seconde cohorte, les métaphores béhavioristes sont moins nombreuses que les métaphores constructivistes à la fin de cette première année de formation. Selon les auteurs, le changement significatif observé dans le second groupe témoigne du potentiel qu'ont les cours à influer sur les croyances, tandis que le maintien des métaphores béhavioristes au sein du premier groupe serait associé à l'expérience de classe, laquelle " efface » les effets des cours préalables (24). Ces auteurs, qui postulent que les enseignants doivent évoluer vers des croyances constructivistes, émettent I'hypothèse que ces étudiants n'ont pas suffisamment bénéficié d'opportunités de réflexion quant à la façon dont les concepts enseignés sont liés aux réalités des classes. Dès lors, ils n'ont pas pu procéder à une articulation en profondeur de leurs théories et de leurs expériences de stage. Selon eux, la pratique réflexive offrirait la possibilité de combler efficacement ce vide entre la théorie et la pratique professionnelle. Nous reviendrons sur cette étude lors de la discussion. 
Doudin, Pfulg, Martin et Moreau (2001) ont étudié les conceptions d'étudiants et d'enseignants à propos de l'intelligence, d'une part, et du rôle de l'enseignant dans les difficultés d'apprentissage et l'échec scolaire, d'autre part. Leur analyse atteste, dans la plupart des cas, une bonne cohérence entre ces deux catégories de conceptions. Elle montre, par ailleurs, qu'étudiants et enseignants se différencient entre eux par leurs profils de réponses. Enfin ces chercheurs observent une évolution des profils de réponses en fonction de l'avancement des étudiants dans le cursus de formation et de l'expérience professionnelle des enseignants en fonction. Dans l'ensemble, les étudiants présentent une conception plutôt socioconstructiviste de l'intelligence ainsi qu'un fort engagement face aux difficultés d'apprentissage et à l'échec scolaire. Ce profil est cependant plus marqué chez les étudiants débutant leur formation et n'ayant pas encore réalisé de stage pratique. Les étudiants ayant réalisé au moins un stage se caractérisent davantage par des conceptions hétérogènes. À l'inverse des futurs enseignants, les enseignants en fonction ont une conception plutôt innéiste de l'intelligence et un engagement modéré par rapport aux difficultés et à l'échec des élèves. Parmi eux, les débutants (un à cinq ans d'expérience) ont un point de vue socioconstructiviste de l'intelligence, mais ne s'engagent pas dans la lutte contre l'échec scolaire. Les plus expérimentés (plus de quinze ans d'expérience) sont les plus cohérents : ils se caractérisent par des conceptions innéistes et ne s'engagent pas non plus. Les enseignants moyennement expérimentés présentent également un faible engagement bien qu'ils se déclarent constructivistes.

Selon Doudin et ses collègues (2001), ces résultats reflètent une séquence développementale quant à l'évolution des conceptions de l'étudiant, puis de l'enseignant durant sa vie professionnelle. Ils identifient trois phases ou expériences clés: d'abord, les stages déstabilisent les conceptions des étudiants; ensuite, l'entrée dans le métier entraîne une absence d'engagement de l'enseignant face aux difficultés de ses élèves et, enfin, la pratique professionnelle favorise la mise en place progressive d'une conception innéiste de l'intelligence. Afin d'expliquer ces changements, notamment en ce qui concerne les stages, ils émettent l'hypothèse d'une confrontation des conceptions socioconstructivistes des étudiants avec les conceptions innéistes des praticiens expérimentés, entraînant un éloignement des théories idéales défendues en formation. Ainsi le contact avec le terrain aurait « un effet déstabilisateur, voire régressif » (Doudin et al., 2001, p. 180). Lors de l'entrée dans la vie professionnelle, le jeune enseignant tend à se conformer aux normes ambiantes: "Le processus de socialisation et d'identification aux conceptions du groupe de professionnels engagé lors des stages se poursuit avec l'entrée dans la pratique " (Doudin et al., 2001, p. 181). En cours de profession, le passage progressif à une conception innéiste de l'intelligence constituerait un refuge pour les enseignants, leur permettant de désigner des « coupables extérieurs " pour expliquer les difficultés ou les échecs des élèves, évitant par là des remises en cause de leur efficacité en tant qu'enseignant. Dit autrement, les croyances innéistes assumeraient une fonction justificatrice, permettant de légitimer une absence d'engagement face aux difficultés des élèves. En conclusion de ces constats et analyses, les auteurs insistent sur l'importance d'actions de formation continue afin d'éviter que les efforts réalisés en formation pour agir sur les conceptions et les pratiques ne soient réduits à néant.

De ces résultats, se dégage une hypothèse formulée de façon plus ou moins explicite par tous les auteurs mentionnés ci-dessus. Les cours dispensés en formation initiale peuvent, dans une certaine mesure, influencer les croyances du futur enseignant, voire les changer. Cependant le contact avec le monde de l'enseignement lors des stages supplanterait l'effet des cours. Quant à l'entrée dans la vie professionnelle, son effet paraît délétère pour les conceptions psychopédagogiques que la formation initiale s'est efforcée de mettre en place. En définitive, il semble 
bien que la formation théorique donnée aux futurs enseignants soit prise en étau entre l'influence persistante de l'expérience scolaire de ceux-ci en tant qu'élèves et les effets cumulés de socialisation provenant des stages, d'abord, de l'entrée dans le métier, ensuite. Ceci expliquerait en définitive l'influence limitée de la formation initiale sur les conceptions des enseignants.

L'étude menée par Hausoul (2005) va dans le sens de cette interprétation des résultats de recherche. Celle-ci a retrouvé, après trois ans d'entrée dans le métier, des enseignants ayant bénéficié, lors de leur dernière année de formation initiale, d'une action de formation intensive visant à les sensibiliser aux effets potentiellement négatifs du redoublement. Hausoul (2005) constate que ceux dont les conceptions en matière de redoublement avaient évolué à la suite de cette formation persistent à douter de l'efficacité de cette pratique pédagogique. Toutefois, ils déclarent autant de décisions effectives de redoublement que leurs condisciples de formation qui ne s'étaient pas laissés convaincre et qui, eux-mêmes, présentent des taux d'échec conformes à la norme particulièrement élevée en Communauté française de Belgique. Bref, si l'action de formation semble avoir eu un effet persistant sur les conceptions de certains enseignants en matière de redoublement, celle-ci ne leur a pas donné les armes pour résister à la « loi du milieu ». En définitive, il faut reconnaître qu'il existe peu de données attestant les effets produits par la formation initiale des enseignants.

\section{Les leçons des études relatives au staff development}

Richardson (1996) soutient que la difficulté de changer les croyances au sein des programmes de formation initiale est liée au manque de pratique des étudiants en ce qui concerne l'enseignement, et donc à la difficulté de les aider à lier leurs croyances à celle-ci. En effet, cette auteure a comparé les résultats des études sur le changement des croyances de futurs enseignants et celles relatives aux enseignants chevronnés. Elle constate que celui-ci est plus aisé à promouvoir et à réaliser effectivement chez les enseignants en fonction, au sein des programmes de développement professionnel, lorsque ces derniers engagent les enseignants à lier leurs croyances et connaissances à leur pratique pédagogique. De plus, toujours selon elle, beaucoup de recherches ont abouti au constat d'un transfert dans leur pratique. Elle émet donc l'hypothèse qu'un facteur majeur expliquant cette différence est lié à l'expérience en tant qu'enseignant: "La connaissance pratique, profonde, tenue par les enseignants expérimentés est fortement liée à l'action, et c'est cette action qui est perçue par les enseignants comme étant le centre d'intérêt du changement. " (Richardson, 1996, p. 114)

Richardson et Placier (2001) reprennent cette thèse et l'examinent systématiquement au regard des études anglo-saxonnes portant sur les effets des démarches visant à promouvoir le développement professionnel des enseignants. Selon ces auteurs, ce champ s'est structuré autour d'une opposition conceptuelle qu'avaient clarifiée Chin et Benne (1969). Dans un ouvrage classique, ces auteurs distinguent trois stratégies d'innovation. La première (qui concerne peu la discussion qui suit) est intitulée "power-coercitive ». Comme son nom l'indique, il s'agit d'imposer un changement par décret ou lois et, plus généralement, d'opérer sur les rapports de force entre les individus et/ou groupes d'individus; les mouvements non violents tels que ceux inspirés par Gandhi sont à ranger dans cette catégorie. Les approches empirico-rationnelles constituent la seconde catégorie. Elles reposent généralement sur le savoir issu des recherches scientifiques qu'il s'agit de disséminer de telle sorte qu'il inspire les actions des praticiens. La démarche est top-down et est enracinée dans une conception rationnelle de l'être humain: si celui-ci connaît la "vérité " scientifique, il s'attachera à tenir compte du savoir nouvellement acquis dans son agir professionnel. Enfin l'approche normative 
rééducative repose sur l'idée que les individus agissent en fonction de normes socioculturelles : tout changement de croyances et/ou de pratiques un tant soit peu important requiert une transformation en profondeur des normes cognitives et comportementales qui implique l'individu dans ses relations avec son entourage social.

Dans le champ qui nous concerne, les approches empirico-rationnelles ont connu une première vague en liaison avec les recherches processus-produit (Crahay, 2006). II s'agissait à l'époque de disséminer les teaching skills, également désignées comme des compétences, dont les recherches empiriques avaient montré l'efficacité en termes d'apprentissage des élèves. Sparks et LoucksHorsley (1990, cités par Richardson \& Placier, 2001) soulignent que ces dispositifs reposent sur un training model qui postule, d'une part, que certains comportements et/ou techniques sont dotés d'une valeur telle qu'ils méritent d'être généralisés et, d'autre part, que n'importe quel enseignant ou élève maître est susceptible de s'approprier et de reproduire ces comportements et/ou techniques. Avec la révolution cognitive dont nous avons rappelé l'importance ci-dessus, les approches empirico-rationnelles se sont étendues aux connaissances et croyances des enseignants. Qu'il s'agisse de modifier des comportements, des connaissances ou des croyances, les interventions de formation relevant de ce courant d'idées sont de courte durée. Par exemple, Sparks (1988, cité par Richardson \& Placier, 2001) analyse les effets d'une intervention comportant cinq séances sur les croyances et pratiques de 19 enseignants au niveau du secondaire inférieur (collège). Autre exemple, Krajcik, Layman, Starr et Magnusson (1991, cités par Richardson \& Placier, 2001) examinent les effets d'une session de formation d'été s'étendant sur trois semaines. Notons que, dans ces études, on observe des effets à court terme sur les croyances et les pratiques de certains enseignants.

L'efficacité à long terme de ces approches n'est pas assurée. En atteste l'étude de Stallings et Krasavage (1986, cités par Richardson \& Placier, 2001) qui porte sur le suivi pendant quatre ans d'enseignants du secondaire inférieur ayant bénéficié d'une formation intensive sur la façon de gérer au mieux le temps scolaire. Initialement, le programme de formation comportait trois modalités. Dans la première, seuls les enseignants chargés de la lecture bénéficient de la formation alors que, dans la seconde, celle-ci englobe, en plus des enseignants chargés de la lecture, ceux responsables des arts et du langage (language arts teachers) et que, dans la troisième, tous les enseignants de l'école sont concernés. De manière générale, cette formation s'avère efficace tant en termes d'implémentation des stratégies comportementales recommandées aux enseignants qu'en termes de réussite des élèves lors de tests de rendement. Par ailleurs, il est significatif de relever que les effets les plus importants sont observés en relation avec la troisième modalité (puis la deuxième et enfin la première). Cette observation conduit Stallings (1989) à souligner l'importance d'impliquer l'ensemble des enseignants d'une école dans les programmes de formation continue si l'on souhaite que celle-ci porte un maximum de fruits. Cependant, trois ans plus tard, les effets de cette formation initialement efficace se sont estompés chez bon nombre d'enseignants.

Un large consensus s'est désormais fait parmi les chercheurs concernant les conditions d'efficacité des stratégies de développement professionnel. On le doit au travail de synthèse de différents auteurs (cf. Richardson \& Placier, 2001, pour une liste). Fullan (1990) les résume comme suit:

- le programme de formation doit impliquer l'ensemble des enseignants de l'école (school wide), favoriser la collégialité et cibler les problèmes rencontrés en son sein (context specific) ;

- le chef d'établissement doit encourager le changement et s'engager dans le processus de travail ; 
- le programme doit s'étendre sur le long terme et reposer sur des ressources budgétaires suffisantes pour permettre l'achat du matériel nécessaire, le remplacement des enseignements lorsqu'ils sont en formation, l'invitation d'experts, etc. ;

- le contenu du programme de formation doit être étayé par des recherches récentes à la méthodologie rigoureuse.

La liberté de s'engager dans le processus de changement est retenue par certains auteurs et non par d'autres. Ces caractéristiques se retrouvent le plus souvent dans les interventions relevant de l'approche normative rééducative. Richardson et Placier (2001) évoquent 12 projets relevant de cette approche. Nous en proposons un tableau synoptique situé en annexe 2. On constate d'emblée que la majorité de ces projets ont trait à l'enseignement des mathématiques ou à celui des sciences. De plus, presque tous sont d'obédience constructiviste. Comme pour les études relatives aux effets de la formation initiale, il faut remarquer que l'ampleur des études est extrêmement variable, allant de 3 enseignants à une quarantaine. De l'examen de ces études, Richardson et Placier (2001) tirent argument en faveur de l'efficacité de l'approche normative rééducative. De façon plus précise, ces deux auteurs soulignent l'importance de plusieurs composantes de cette approche, dont la conjugaison leur paraît déterminante (25) :

- les enseignants doivent commencer à appréhender leurs croyances ou présupposés implicites. Ce processus d'appréhension est favorisé par le dialogue, en particulier avec ceux qui comprennent ce qu'est l'enseignement et connaissent le contexte particulier dans lequel les praticiens œuvrent ;

- le dialogue au sein de la communauté des enseignants (community dialogue) constitue semble-t-il l'élément clé (voir à ce propos McLaughlin, 1994), particulièrement au début du processus évolutif. À cet égard, la confiance au sein de la communauté est cruciale : l'enseignant doit se sentir en confiance pour pouvoir mettre sur la table ses problèmes, discuter de ses pratiques inefficaces et accepter sa responsabilité (au moins, partielle) dans les dysfonctionnements observés ;

- dans une phase ultérieure, il est important que l'enseignant mette en question ses croyances, s'interroge sur leur origine, leur motif et leur validité. II faut qu'il considère qu'il peut en changer. Lorsque l'enseignant en est à ce stade, la discussion avec un autrui significatif (un ami critique) peut s'avérer féconde. Celui-ci peut offrir un autre langage qui aide à dévoiler les croyances implicites et ouvre de nouvelles perspectives de réflexion sur la pratique.

\section{DISCUSSION}

\section{En définitive, les croyances et connaissances des enseignants sont-elles modifiables?}

Bien que de nombreuses recherches dans le domaine reposent sur des méthodes incertaines et présentent des données fragiles, une réponse positive peut être donnée à cette question essentielle. II convient cependant d'ajouter d'emblée que ce processus ne va pas de soi. Autrement dit, il requiert certaines circonstances que nous serions tentés de qualifier d'exceptionnelles. La thématique, largement traitée dans la littérature, de résistance des enseignants au changement ou de leur imperméabilité aux processus de formation atteste la difficulté de modifier les croyances et connaissances des enseignants.

La thèse défendue par Richardson et Placier (2001) en soi interpelle. De leurs revues de la littérature, ces auteurs concluent que les croyances et connaissances 
des enseignants ne changent pas, ou alors rarement, des suites de la formation initiale. En revanche, des évolutions sont observables chez les enseignants en fonction, soit de façon «naturelle ", c'est-à-dire indépendamment de tout processus de formation in service, soit lorsque ces formations s'inscrivent dans une approche de type normative rééducative. Or, à bien examiner les données des études rapportant des évolutions dites naturelles (naturalistic change), celles-ci n'ont rien de naturel. Le développement remarquable de Kerrie Baughman a été accompagné au quotidien par la sollicitude de son observateur personnel (Bullough \& Baughman, 1997). Quant à Russell (1995), il s'est mis à l'épreuve de la réalité afin de montrer le bien-fondé de ses convictions de formateur. Bien sûr, il ne s'agit pas d'ignorer ces études remarquables par ailleurs ; il convient d'en souligner le caractère exceptionnel et donc le caractère non généralisable au commun des enseignants. Quant aux études relevant du staff development, tous les auteurs finissent, d'une manière ou d'une autre, par indiquer la difficulté de faire évoluer les croyances et connaissances des enseignants. Ceci requiert des conditions que Fullan (1990) a remarquablement synthétisées et celles-ci doivent - pensonsnous - être complétées par celles distinguées par Richardson et Placier (2001) concernant les approches normatives rééducatives. À ce stade de la discussion, il nous paraît important de pointer deux de ces conditions: l'évolution des croyances et connaissances s'opère d'abord sur le long terme et ensuite lorsque l'ensemble des enseignants de l'école sont impliqués et, mieux encore, lorsqu'ils constituent une communauté de dialogue.

Rares sont les chercheurs qui pensent qu'il est possible de faire évoluer les croyances des enseignants sur le court terme. Ceux-là - nous pensons à Guskey (1986), Mevarech (1995), Prawatt (1992) et Sparks (1988) - s'inscrivent dans l'approche empirico-rationnelle du changement; d'autre part, ces chercheurs sont avant tout concernés par l'implémentation de pratiques nouvelles en rapport soit avec la pédagogie de la maîtrise (Guskey, 1986), soit avec l'enseignement des mathématiques (Prawatt, 1992; Sparks, 1988), soit avec les deux (Mevarech, 1995). Bien plus, ces chercheurs soutiennent qu'il est plus efficace de changer les pratiques avant de changer les croyances. Ce point de vue n'est pas partagé par Richardson et Placier (2001) qui rappellent l'étude de cas réalisée par Richardson, Anders, Tidwell et Lloyd (1991) : l'enseignante observée a modifié en profondeur ses croyances concernant la compréhension en lecture avant de transformer sa pratique d'enseignement. Cette problématique est cruciale. Malheureusement, nous n'avons pas trouvé d'études qui la prennent à bras-le-corps et y apportent une ou des réponse(s) claire(s) (26). La difficulté d'induire des changements dans les croyances des enseignants et, corrélativement, dans leurs pratiques mérite qu'on s'interroge sur les raisons de cette résistance aux démarches de formation.

\section{Quelques hypothèses concernant la relative imperméabilité des croyances des enseignants aux processus de formation}

Nombreux sont les auteurs qui évoquent l'étude de Lortie (1975) pour expliquer la résistance des croyances initiales des élèves maîtres au processus de formation. Ainsi en est-il de Bullough et Knowles (1990, 1991), de Knowles (1992), de Borko et Putnam (1996), de Richardson et Placier (2001) et beaucoup d'autres encore. À ce propos, Bullough et Knowles $(1990,1991)$ ont mené une étude longitudinale de l'identité professionnelle de sept étudiants durant leur première année de formation. En se basant sur les données relatives à cinq d'entre eux, Knowles (1992) montre que les modèles les plus influents dans la construction de leur image d'eux-mêmes, en tant qu'enseignants, sont les modèles qui sont intervenus tôt dans leur scolarité. L'auteur n'a relevé que très peu d'impact des expériences 
vécues au cours de la formation sur ce processus identitaire, de même que dans leurs comportements durant les stages. Par ces conclusions, Knowles (1992) conforte la thèse de Lortie (1975) selon laquelle les milliers d'heures passées désormais par tout un chacun sur les bancs de l'école durant toute la scolarité influencent fortement la construction des croyances des futurs enseignants, mais aussi - peut-on supposer - celles des parents et de tout individu ayant été scolarisé. Ces croyances construites précocement ont tendance à se perpétuer et seraient réfractaires au changement.

Pour étayer cette idée d'une influence précoce des modèles rencontrés dans les salles de classe, Pajares (1992, p. 322) cite Van Fleet (1979) qui décrit une visite chez son frère où il découvre sa nièce âgée de huit ans en train de "jouer l'enseignante ". Celle-ci, décrit le chercheur, était dans sa chambre, entourée de ses poupées et plantée devant son tableau noir : "Se tenant là avec la craie qui se balançait à la perfection entre ses doigts, avec la bonne intonation et l'expression du visage adéquate, elle enseignait, exhortant ses poupées élèves à être particulièrement attentives durant cette leçon importante " (Van Fleet, 1979, p. 283). Et Pajares (1992) de commenter : «La petite demoiselle Van Fleet était en train de développer un ensemble de croyances et de pratiques liées au fait d'être un enseignant. Quel que soit l'adulte qu'elle pourrait devenir, pendant au moins les dix prochaines années, elle allait aiguiser ses pratiques et renforcer ses croyances bourgeonnantes, si celles-ci ne sont pas déjà enracinées. » (Pajares, 1992, p. 322)

Ce positionnement théorique, défaitiste, fait sens. Car il est communément admis que plus tôt une croyance est incorporée dans la structure cognitive d'un individu, plus il sera difficile de l'y déloger. À l'opposé, les croyances nouvellement acquises sont plus vulnérables au changement. Ainsi, selon Nisbett et Ross (1980), il faut compter avec un effet de primauté suivant lequel les croyances construites à partir des expériences du jeune âge influencent les interprétations et les perceptions ultérieures. Avec le temps, les individus se construisent ainsi des théories personnelles robustes qui persistent, même face à des explications contradictoires logiques, convaincantes, ou encore scientifiquement fondées. Des phénomènes de persévérance s'installent, reposant sur divers processus cognitifs tels que la perception sélective des informations, voire leur déformation (Schommer, 1990) : les éléments soutenant les croyances antérieures sont pris en compte, tandis que les éléments conflictuels sont ignorés ou déformés. Les croyances influenceraient aussi la mémoire: les individus se remémorent certains événements (souvenirs), en les déformant si nécessaire de façon à soutenir leurs croyances (Nisbett \& Ross, 1980).

En conformité avec cette théorie générale concernant le fonctionnement des croyances, il est logique de penser que l'éventail des croyances que les étudiants se sont forgées au fil de leurs expériences en tant qu'élèves est solidement ancré au moment où ils entrent en formation initiale d'enseignants (Pajares, 1992 ; Strauss, 1996, cité par Woolfolk Hoy \& Murphy, 2001 ; Richardson, 1996 ; Wilson, 1990). Par ailleurs, reconnaître la solidité de l'ancrage des croyances initiales n'implique pas ipso facto qu'elles soient indéracinables : certains auteurs semblent faire, le plus souvent implicitement, le pas. Peut-être convient-il de reconnaître à la fois l'enracinement solide de ces croyances et la nécessité de dispositifs de formation s'attaquant de front à ces croyances qu'il paraît souhaitable de modifier. À cet égard, Richardson (1996) fait remarquer que les connaissances psychopédagogiques instituées sont présentées aux futurs enseignants selon un processus d'apprentissage explicite, alors que leurs croyances initiales sont de nature implicite, sédimentées depuis l'enfance par la conjonction d'apprentissages vicariants et d'interactions verbales au cours desquelles les croyances psychopédagogiques de sens commun sont renforcées. 
Par ailleurs, se référant à la notion de centralité de certaines croyances par rapport à d'autres, notion développée par Rokeach (1976) notamment, Richardson (1996) émet l'hypothèse que certaines croyances psychopédagogiques, les plus centrales, seraient plus résistantes au changement que d'autres. Présentant davantage de connexions avec les autres croyances du système, ces croyances ou conceptions centrales requerraient davantage d'efforts pour évoluer. Ainsi il conviendrait de considérer que les changements de croyances à court ou à long terme sont liés à la centralité. Rokeach (1976) formule l'hypothèse que "les changements induits au niveau des croyances centrales ont des répercussions relativement plus grandes sur le reste du système de croyances que des changements induits au niveau de croyances moins centrales " (p. 55).

De notre point de vue, la réflexion théorique de Richardson (1996) relance le débat. Sur le plan de la recherche, cela implique des études sur la structuration des croyances des enseignants. Selon le modèle conceptuel de Rokeach (1976), repris par Richardson (1996), certaines croyances seraient plus centrales que d'autres. Plus généralement, ce modèle conceptuel suggère une structuration des croyances en strates superposées: il y aurait des couches superficielles et profondes. À cette conception s'oppose celle de Green (1971) qui suggère que les personnes peuvent organiser leurs croyances en clusters (grappes, ensembles de croyances) plus ou moins isolés les uns des autres, voire totalement dépourvus de relations entre eux. Ainsi les enseignants peuvent maintenir, implicitement ou non, des croyances pouvant apparaître contradictoires aux yeux des chercheurs. Par exemple, Van Driel, Bulte et Verloop (2007) ont étudié les relations entre les croyances d'enseignants du secondaire à propos, d'une part, de l'enseignement-apprentissage et, d'autre part, des objectifs que devrait poursuivre le programme officiel de l'éducation à la chimie. Ils ont discriminé les répondants en fonction de leurs structures de croyances. Quatre groupes d'enseignants ont été identifiés, dont le plus large $(45 \%$ des participants) combine deux orientations contradictoires quant à l'enseignementapprentissage et aux objectifs du programme officiel: une orientation relative à l'enseignement-apprentissage centrée sur la matière, et la seconde relative au programme et caractérisée par une opposition à l'idée selon laquelle les concepts théoriques doivent être enseignés en priorité. Toujours selon ces auteurs, "certains enseignants combinent délibérément différentes idées à propos de l'enseignement car ils ont trouvé que ces différentes perspectives pouvaient être utiles dans différentes situations d'enseignement. D'autres, apparemment, ignorent les différences et ont parfois des incohérences dans leurs systèmes de croyances " (Van Driel, Bulte \& Verloop, 2007, p. 169). La recherche d'Issaieva et Crahay (2010), portant notamment sur les conceptions évaluatives d'enseignants français de la fin de l'enseignement primaire, aboutit à des constats analogues : les enseignants combinent de façon plus ou moins cohérente des croyances favorables à une approche formative de l'évaluation avec d'autres relevant d'une perspective normative de celle-ci.

La question de la structuration des croyances nous paraît cruciale et, par ailleurs, largement sous-explorée. Des connaissances validées à ce propos seraient utiles tant pour concevoir des programmes de formation que pour interpréter les résultats des recherches à ce sujet. Actuellement, on place sur un même plan toutes les études qui ont tenté d'induire un changement de croyances. Si la thèse défendue par Rokeach (1976) et reprise par Richardson (1996) est exacte, les succès et échecs de formation ne devraient pas être tous considérés de la même manière. Ainsi, selon cette hypothèse théorique, les formations visant des changements de croyances de surface devraient atteindre plus aisément leurs objectifs, mais les effets obtenus devraient être davantage susceptibles de s'estomper, même à court terme. À l'inverse, les formations visant la transformation de croyances centrales devraient rencontrer davantage de résistances en 
cours de processus; une fois engendrés, les effets devraient se maintenir sur le long terme et se répercuter sur d'autres croyances. Si, par contre, c'est le modèle de Green qui s'approche le plus de la réalité psychologique, la transformation des croyances peut être abordée à peu près par n'importe quel bout; il s'agirait seulement de tenir compte des paquets de croyances qui s'agglutinent en clusters. En définitive, nous plaidons pour que l'agenda des recherches futures réserve une place importante à l'étude de la structuration des croyances.

Enfin il paraît nécessaire de prendre en compte ce que sont les préoccupations majeures des enseignants en formation. Les recherches portant sur les stades de développement des enseignants sont unanimes et explicites sur ce point: leur préoccupation première est la gestion de la classe. Les enseignants en formation et débutants veulent faire leurs preuves face à une salle de classe (cf. notamment Fuller \& Bown, 1975). On peut en inférer que les théories de l'apprentissage et du développement psychologique des enfants ne sont pas au cœur des intérêts premiers des enseignants à ce moment de leur évolution. De ceci découle une hypothèse, en soi banale, basée sur l'idée d'une succession «obligée " des transformations à opérer chez les enseignants : certains changements de croyances devraient nécessairement en précéder d'autres. À l'appui de cette hypothèse, on convoquera Hollingsworth (1989) qui, observant comment des étudiants en formation s'approprient l'enseignement de la lecture, constate que ceux-ci doivent maîtriser les techniques de conduite de la classe avant de pouvoir travailler leurs croyances pédagogiques et, tout particulièrement, les croyances pédagogiques de contenu.

\section{Les croyances initiales des enseignants : un filtre à prendre en compte dans la conception des formations}

Des recherches (Hollingsworth, 1989 ; Kagan, 1992 ; Borko \& Putnam, 1996 ; Richardson, 1996 ; Richardson \& Placier, 2001) ont montré l'influence des croyances dans le processus de formation initiale. Car, bien évidemment, les futurs enseignants ne débutent pas leur formation ex nihilo. Comme montré largement ci-dessus, ceux-ci possèdent des croyances qui influenceront non seulement leurs pratiques, mais également ce qu'ils apprendront et la manière dont ils l'apprendront. Or, en vertu du mécanisme bien connu en psychologie de la perception sélective des informations (Tversky \& Kahneman, 1974), le futur enseignant interprète et évalue ses expériences de formation à travers le prisme de ses croyances, qu'il s'agisse des cours théoriques, des pratiques recommandées ou de ses expériences de stage. Par exemple, Feiman-Nemser et Buchmann (1989) présentent le cas d'une étudiante qui, influencée par ses croyances initiales, ne retient pas, lors des lectures recommandées, les éléments d'information qui vont à l'encontre de ces dernières. Cette étudiante va jusqu'à modifier - inconsciemment le message véhiculé par le texte pour qu'il corresponde à ses croyances. D'une manière générale, Feiman-Nemser et Buchmann (1989) suggèrent que les textes présentés aux étudiants et, plus largement, tout enseignement peut produire un effet inverse à celui recherché ou attendu par les formateurs, dans la mesure où tout étudiant interprète les informations qui lui sont présentées en utilisant ses croyances, s'efforçant avant tout de conserver voire de renforcer ces dernières (27).

Ross, Johnson et Smith (1991, cités par Borko \& Putnam, 1996, et par Richardson, 1996) ont étudié l'influence des croyances et connaissances des futurs enseignants sur ce qu'ils peuvent apprendre en formation. Leur programme suivi, appelé Proteach, était axé spécifiquement sur le développement de capacités réflexives et d'une base de connaissances relatives à l'enseignement. Ayant mené plusieurs études de l'impact des activités de formation sur les réflexions et les 
pratiques des étudiants, ces chercheurs concluent que, parmi les multiples facteurs qui influencent l'apprentissage de l'enseignement, les croyances à l'entrée en formation sont l'un des plus significatifs. De même, Hollingsworth (1989), étudiant l'impact d'un programme de formation d'orientation constructiviste sur les croyances relatives à l'apprentissage, observe que les changements produits sont fonction des croyances initiales des étudiants et que ceux qui étaient davantage aptes à confronter celles-ci aux théories enseignées ont construit des connaissances plus profondes.

Les croyances des enseignants remplissent donc bien une fonction épistémique. Plus particulièrement, elles sont susceptibles d'opérer comme un filtre à travers lequel les phénomènes, les informations sont sélectionnés et interprétés. La littérature relative à l'introduction de pratiques innovantes ou à l'utilisation de nouveaux programmes (par exemple, Borko \& Putnam, 1996 ; Olson, 1980, 1981, cités par Clark \& Peterson, 1986 ; Richardson, 1990) illustre particulièrement bien ce mécanisme. Ainsi, dans le cadre d'un programme de recherche, la California study of elementary mathematics, portant sur le changement des pratiques des enseignants suite à de nouvelles directives nationales en matière d'enseignement, Cohen (1990) présente le cas d'un enseignant. Ce professeur répartissait ses élèves en petits groupes pour répondre à la demande de son État, c'est-à-dire de pratiquer plus d'enseignement coopératif. Cependant, si l'organisation de la classe était modifiée physiquement, le contenu et le contexte des interactions en classe n'avaient pas changé. L'enseignant interagissait avec les élèves individuellement ou avec la classe entière, alors qu'il aurait dû encourager les interactions directes entre élèves. Ces dernières étaient même découragées. En définitive, la façon dont l'enseignant interprétait et exécutait les nouvelles directives passait par le filtre de ses croyances relatives aux interactions avec les élèves. Ainsi ce type de résultats suggère que, d'une part, avant toute innovation, il est essentiel d'examiner ces filtres et que, d'autre part, des changements au niveau des pratiques doivent être accompagnés de changements au niveau des croyances (Clark \& Peterson, 1986 ; Borko \& Putnam, 1996 ; Van Driel, Bulte \& Verloop, 2007).

Reconnaissant l'importance des croyances initiales des enseignants en formation, de nombreux auteurs (parmi lesquels Cronin-Jones, 1991; Fenstermacher, 1979 ; Pajares, 1992 ; Olson, 1993 (28) ; Richardson, 1996) estiment qu'il est primordial de les rendre explicites, avant d'avoir pour objectif d'essayer de les modifier. En ignorant le poids des conceptions initiales, le formateur court le risque de voir se perpétuer des conceptions et des pratiques d'enseignement obsolètes ou inefficaces. Ce point de vue est partagé par de nombreux auteurs. II a été clairement explicité par Kagan (1992). Cette auteure rapproche les observations évoquées ci-dessus de ce que l'on connaît des conceptions des élèves dans le domaine des sciences et des mathématiques. En conséquence, elle propose d'adapter le cadre théorique du conceptual change à la problématique de la transformation des croyances des enseignants. Ceci l'amène à recommander une démarche d'intervention en trois temps :

- aider les étudiants à prendre conscience de leurs croyances implicites et, partant, à les expliciter ;

- les confronter avec des éléments pouvant déboucher sur une mise en question de leurs croyances en mettant en évidence leurs contradictions internes, leurs incohérences, voire leurs inadéquations par rapport à la réalité de l'enseignement ;

- donner l'opportunité aux étudiants d'intégrer de nouvelles connaissances, de sorte de différencier les anciennes croyances, voire de remplacer les conceptions totalement inadaptées (29).

Bref, il s'agirait de susciter un processus de déséquilibration/rééquilibration tel que théorisé par Piaget (1975) dans son ouvrage L'équilibration des structures cognitives. 


\section{Existe-t-il de meilleures croyances que d'autres?}

La voix de la déséquilibration (ou du conceptual change) est toutefois problématique en ce qui concerne les croyances des enseignants, car cette démarche postule qu'il existe des connaissances plus valides que d'autres, d'où la possibilité de générer des équilibrations majorantes (Piaget, 1975). Or, comme le souligne Kagan (1992) elle-même, l'enseignement est un domaine où les connaissances assurément valides sont rares. Comment, dès lors, conduire un processus de transformation des croyances des enseignants si l'on n'a pas l'assurance de les orienter vers un mieux-penser et/ou un mieux-agir ? Bien plus, pourquoi entreprendre ce travail de formation?

Examinant les approches qui s'inscrivent dans le courant du praticien réflexif (cf. Schön, 1994), Liston et Zeichner (1989, cités par Kagan, 1992) distinguent trois positions. À un extrême, on trouve les approches qui considèrent que toutes les croyances se valent; le processus réflexif se donne alors pour objectif d'élucider les préférences, mais il n'est pas question de juger les croyances des uns et des autres. À l'autre extrême, on trouve les dispositifs de formation qui considèrent que les croyances et connaissances des enseignants doivent in fine se conformer à un ensemble de standards professionnels, ceux-ci définissant les normes du métier, qui sont elles-mêmes le produit d'un consensus parmi les experts. Entre ces deux extrêmes, on trouve des approches qui visent à ce que les enseignants soient au clair avec le type de courant pédagogique auxquels ils adhèrent (socioconstructivisme, enseignement transmissif, etc.) et s'attachent à affermir leur cohérence propre, quel que soit le courant choisi.

Peu d'auteurs soulèvent ces questions pourtant centrales, qu'il s'agisse de définir la finalité d'une formation ou d'évaluer les changements de croyances. Or, dans la plupart des études recensées ci-dessus, l'évolution des croyances est assimilée à un processus de substitution : au début de la formation, l'enseignant pensait $A$; à la fin, il pense $B$. Cette façon de poser le problème a tout son sens dès lors que l'on est assuré que penser $B$ est susceptible d'engendrer de meilleures pratiques qu'en pensant $A$. Mais il faut reconnaître qu'en éducation, nous sommes rarement dans ce cas de figure. Même parmi les chercheurs en sciences de l'éducation, il est rare qu'il y ait unanimité de croyances. Examinons quelques cas précis et discutons-les.

Dans l'étude de Leavy et ses collègues (2007) déjà présentée ci-dessus, les étudiants qui ont réalisé des stages sur le terrain retrouvent en fin de formation les croyances (appréhendées à travers l'expression de métaphores) prévalant en début d'année. À l'opposé, les étudiants qui bénéficient uniquement de cours théoriques pendant le même laps de temps privilégient les métaphores reflétant des convictions socioconstructivistes. Pour les auteurs, il va de soi que les évolutions positives prennent la direction du socioconstructiviste. Ne remettant nullement en cause ce postulat, les chercheurs incriminent le processus de formation et, plus spécifiquement, le manque d'opportunités de réflexion quant à la façon dont les concepts enseignés sont liés aux réalités des classes. Cette hypothèse est recevable, mais on peut en avancer une concurrente : confrontés aux difficultés inhérentes à toute gestion de classe, les jeunes enseignants expérimenteraient l'aspect pratique des principes béhavioristes ainsi que leurs effets à court terme et, partant, les intégreraient à leur pratique quotidienne. Car, il faut l'admettre, nul n'a aujourd'hui fourni la preuve irréfutable de la supériorité du socioconstructivisme sur le béhaviorisme en termes de gestion de la classe. Au contraire, certains chercheurs contestent l'efficacité de ce qu'ils considèrent comme une doxa (cf. notamment Kirschner, Sweller \& Clark, 2006). 
Les croyances des enseignants de culture francophone en ce qui concerne le redoublement sont résistantes aux tentatives entreprises pour les déstabiliser (Crahay \& Donnay, 2001). Bien plus, l'étude menée par Hausoul (2005) a montré que des enseignants qui, au cours de leur formation initiale, avaient changé de croyances, peuvent conserver ces nouvelles croyances après trois années de métier, tout en pratiquant le redoublement comme leurs collègues. Or, dans le cas présent, on dispose de preuves quasi expérimentales de l'absence d'effets positifs du redoublement (Crahay, 2004). Hélas, les enseignants n'ont pas l'occasion d'expérimenter ce qu'auraient été les effets d'une promotion des mêmes élèves sans redoublement (30) ; ils se laissent impressionner par les progrès des redoublants et, n'ayant pas les moyens d'en relativiser l'importance, ils persévèrent à attribuer une trompeuse efficacité à une pratique peu opérante. De surcroît, l'attachement des enseignants au redoublement est en partie de nature sociale. La tradition et le consensus quasi unanime des enseignants, des parents, des responsables et même des élèves pèse lourdement dans la balance : il faut être un héros pour braver cette conviction de sens commun et l'on comprend dès lors aisément l'opportunité de faire porter - comme le recommande Fullan (1990) - le programme de formation sur l'ensemble des enseignants de l'école... sans être sûr que cela suffise. Car, comme l'argumente Draelants $(2006,2009)$, le redoublement fait preuve d'une fonctionnalité certaine dès lors que l'objectif est d'obtenir des classes homogènes en termes de performance d'élèves et que la culture scolaire dominante soutient que des sélections et des orientations doivent être faites. En définitive, les preuves apportées par les chercheurs concernant l'absence d'effets du redoublement eu égard aux apprentissages des élèves ont bien peu de poids dans ce contexte. Pour se légitimer, les enseignants déclarent alors: "Bien sûr, en théorie, le redoublement, ce n'est pas la solution, mais en pratique, c'est autre chose ! »

Le cas de l'enseignement de la lecture fournit un autre exemple où ce qu'il est bon de croire est soumis à débats perpétuels. Bien sûr, la recherche en psychologie cognitive de la lecture (Martinet \& Rieben, 2010) a largement démontré l'importance de la conscience phonologique et de la maîtrise du principe alphabétique. La place à réserver à l'apprentissage du code, la façon de conduire cet apprentissage et l'équilibre à assurer avec les activités mobilisant la recherche de sens restent objets de controverses, parfois vives si l'on en juge ce qui se passe régulièrement en France à propos de ces questions. Manifestement, concernant l'apprentissage de la lecture, la question des bonnes croyances est au moins en partie de nature idéologique. C'est aussi le cas de la composition des classes. La recherche montre de façon convaincante, pensons-nous (Crahay, 2000 ; Crahay \& Monseur, 2006 ; Monseur \& Crahay, 2008), les effets positifs de la mixité sociale des classes et du mélange des élèves forts et faibles ; cette question reste objet de vives polémiques. Seuls l'enseignement des mathématiques et celui des sciences semblent actuellement échapper aux controverses largement médiatisées. C'est dans ce domaine que les recherches anglo-saxonnes font état du plus grand nombre de succès en termes d'évolution des croyances dans le sens du constructivisme.

En définitive, le modèle du changement conceptuel paraît bien trop étroit pour rendre compte des transformations de croyances qu'il paraît souhaitable de susciter dans le cadre de la formation des enseignants. Ce modèle est bien trop strictement cognitif pour résoudre les problèmes qui se posent aux formateurs d'enseignants. II réduit la problématique à des questions de faits (ou de vérité empiriques), ignorant de facto que l'éducation pose aussi des questions de droit (cf. à ce propos Crahay, 2000). II sous-estime également les phénomènes de mise en pratique ainsi que ceux de désirabilité et de consensus social. Ce dernier 
problème est résolu par les approches normatives rééducatives : le consensus se négocie au sein de l'école, entre enseignants, au travers de ce que McLaughlin (1994) nomme la communauté de dialogue. Malheureusement, cette solution ne paraît pas transférable telle quelle au niveau de la formation initiale des enseignants.

II convient, par ailleurs, de noter que bon nombre d'enseignants méconnaissent les résultats des recherches en éducation. Leurs croyances s'élaborent donc le plus souvent indépendamment de ce savoir. Ce constat d'expérience soulève plusieurs questions qui mériteraient d'être étudiées par les chercheurs. Premièrement, il serait intéressant de mieux comprendre comment les praticiens intègrent des savoirs validés empiriquement à leurs croyances spontanées. Cette confrontation de savoirs aux origines et aux statuts différents suscite-t-elle chez eux une réflexion de nature épistémologique sur la validité des croyances ? Plus généralement, lorsque les enseignants sont mis en déséquilibre concernant leurs croyances, comment traitent-ils cette perturbation et in fine comment et pourquoi optent-ils pour telle croyance plutôt que telle autre ? Distinguent-ils les questions de droit des questions de fait ? Autrement dit, y a-t-il jugement d'ordre épistémique de leur part (cf. à ce propos la note de synthèse de Crahay \& Fagnant, 2008) ou leur choix repose-t-il sur un processus d'une autre nature ? Dans un article intitulé The practicality ethic in teacher decision-making, Doyle et Ponder (1977) ont défendu l'idée que les enseignants choisissaient leurs stratégies d'enseignement en fonction de leur caractère pratique plutôt qu'en fonction de leur efficacité ou de leur validité théorique. Qu'en est-il dès lors que l'on interroge les enseignants sur ce qu'ils considèrent comme une croyance juste ? Bref, il nous semble que des recherches pourraient et même devraient porter sur la façon dont les enseignants établissent, lorsqu'ils sont poussés à le faire, ce qu'est une croyance valide ou, au moins, pertinente.

\section{CONCLUSION : L'ÉVOLUTION DES CROYANCES, DES PROCESSUS MULTIPLES}

L'examen des recherches portant sur les effets de la formation initiale et du staff development dévoile, pour la plupart des chercheurs, une conception étriquée de l'évolution des croyances. Dans une majorité d'études, seuls les changements de type substitution de croyances (c'est-à-dire lorsque l'enseignant passe d'une croyance A à une croyance $B$ ) semblent être envisagés. À la lecture de la réflexion conceptuelle de Leuchter (2009), on est amené à considérer qu'il y a d'autres types de changement et que la substitution de croyances n'est probablement pas le changement qui occupe le plus le champ mental des enseignants. Pour Leuchter (2009), rappelons-le, les croyances des enseignants se déploient et évoluent sur quatre continuums allant du systématique au situé, de l'explicite à l'implicite, du déclaratif au procéduralisé, et du scientifiquement fondé aux éléments basés sur l'expérience personnelle et professionnelle. Cette conceptualisation laisse envisager des modalités de changement multiples, complémentaires à la substitution de croyances.

À travers les livres et les manuels, les enseignants sont mis en contact avec des connaissances et des croyances disponibles pour l'ensemble de la communauté savante. Dans la mesure où ils les assimilent, ils se dotent de connaissances et de croyances scientifiquement fondées. Qu'elles soient disciplinaires, psychologiques ou pédagogiques, ces connaissances et croyances provenant de la recherche sont systématisées, transparentes, accessibles et communicables. Le répertoire de connaissances et de croyances professionnelles des enseignants repose en partie sur ces savoirs au caractère relativement stable et consensuel, puisqu'elles ne sont pas soumises aux variations individuelles (Leuchter, 2009). Ces savoirs peuvent devenir fonctionnels à condition d'être ancrés dans les situations et les 
cas issus de l'expérience de l'enseignant. Suivant en cela les leçons de la psychologie cognitive, Leuchter (2009) souligne que ce n'est pas le savoir systématique tel quel qui gouverne l'acte d'enseignement: les connaissances déclaratives doivent être procéduralisées. II faut considérer qu'il y a de la part de tout enseignant un travail, largement inconscient, de restructuration de ces savoirs systématisés, engrangés pour l'essentiel au cours de la formation initiale. Selon cette chercheuse, cette restructuration s'effectue sur la base d'expériences accumulées lors des situations d'interactions. Cette réorganisation entraîne un éloignement des connaissances et croyances des enseignants par rapport aux savoirs systématiques et un rapprochement par rapport aux spécificités situationnelles. Cette «procéduralisation " des connaissances formelles peut aussi être désignée par le terme d'enactement, dans le sens où les connaissances formalisées au niveau de la recherche muent pour "s'encapsuler " dans les pratiques des enseignants.

Le passage du déclaratif au procéduralisé est donc aussi une mutation du scientifiquement fondé aux éléments basés sur l'expérience personnelle et professionnelle. C'est aussi un passage du systématique au situé. Leuchter (2009) rappelle avec force qu'une caractéristique importante du savoir des enseignants chevronnés est d'être situé, c'est-à-dire ancré dans des cas particuliers. Selon elle, les enseignants mettent divers éléments de savoirs et de convictions en réseau autour de cas spécifiques, et non autour d'éléments théoriques. À l'appui de cette idée, elle évoque les recherches qui montrent que le savoir professionnel de l'enseignant se transforme en savoir d'expérience à mesure de l'augmentation de son temps d'enseignement (Gruber, 1999 ; Rumpf \& Kranich, 2000 ; Terhart, 2001). C'est ce type de transformation que subirait le savoir disciplinaire des enseignants. Celui-ci est une partie importante du répertoire cognitif des enseignants. Cependant, au fur et à mesure que l'enseignant pratique, l'organisation de ce bagage se base de plus en plus sur les situations et cas spécifiques qui restructurent le savoir systématique en savoir situationnel (Leuchter, 2009). De plus, les enseignants s'efforceraient de mettre leurs savoirs disciplinaires en relation avec leurs savoirs pédagogiques généraux, constituant ainsi les pedagogical content knowledge conceptualisés par Shulman (1987).

Progressivement, les savoirs explicites se transforment également en savoirs tacites. Les savoirs tacites, qui ont une influence puissante sur la réflexion et l'action des enseignants, seraient constitués, d'une part, de la concaténation de savoirs explicites et, d'autre part, de savoirs expérientiels engrangés souvent de manière inconsciente par les individus. Les savoirs des enseignants se constitueraient donc à la fois à partir d'une "procéduralisation » de connaissances déclaratives, mais aussi à partir d'apprentissages implicites. Rappelons ici également que l'une des caractéristiques du savoir tacite est sa forte persistance, notamment en ce qui concerne la fidélité que les enseignants ont vis-à-vis de leurs croyances qu'ils ne modifient que rarement. Une autre de ses caractéristiques est sa densité, c'est-à-dire le fait qu'il renferme un grand nombre d'éléments de croyances et de connaissances (Leuchter, 2009).

Cette transformation du savoir explicite en savoir expérientiel tacite et procéduralisé aboutit, toujours selon Leuchter (2009), à des hiérarchies de règles de production. Ces savoirs procéduralisés sont utilisés quand, dans une situation donnée, divers indices sont considérés par l'enseignant comme appelant leur application. Ils peuvent être agglomérés ou mis en réseau pour constituer des règles de production supérieures par un processus de chunking. Ce processus, qui facilite le rappel et l'application des règles de conduites en situation, peut être considéré comme une densification du savoir de l'enseignant ayant comme conséquence d'accélérer le rythme des conduites d'enseignement et de prise de décision 
(Bromme, 1992). Leuchter (2009) rapproche cette densification du savoir des concepts de script et de schème, développés dans le cadre des recherches relevant du teacher thinking. Pour elle, le savoir procéduralisé se manifeste dans les conduites en action de l'enseignant, mais fait aussi partie de son processus de perception en situation. L'émergence de savoir procéduralisé peut se voir comme un apprentissage de règles pertinentes pour le processus d'enseignement et qui sont accolées les unes aux autres. Avec l'expérience, ces règles s'enrichissent progressivement d'éléments spécifiques aux situations d'enseignement et deviennent, de ce fait, des ensembles denses de stratégies d'analyse et de fonctionnement qui, en action, permettent une simplification de l'activité d'enseignement ainsi qu'une accélération du processus de pensée interactive des enseignants (Leuchter, 2009).

Le savoir basé sur l'expérience finirait par constituer un système de convictions. Fortement marquées par des aspects émotionnels et des souvenirs personnels ainsi que difficilement exprimables, ces cognitions, qui peuvent se passer de la preuve, sont soumises à un système de forces: on peut être plus ou moins convaincu de leur exactitude ou de leur pertinence (Thompson, 1992). Elles peuvent également être constituées d'éléments contradictoires que les enseignants équilibrent pour évoluer dans la situation d'enseignement-apprentissage (Baumert \& Kunter, 2006 ; Borko \& Putnam, 1996 ; Calderhead, 1996 ; Conners, 1978 ; Cornett, 1990 ; Freeman, 1991 ; Gastager, 2003 ; Halkes \& Deijkers, 2003 ; Haritos, 2004 ; Lampert, 1986 ; Leuchter, 2009 ; Marland, 1977 ; Sardo-Brown, 1990 ; Sinatra \& Kardash, 2004 ; Tomanek, 1994 ; Wagner, 2003 ; Wanlin \& Crahay, soumis ; Wanlin, Lafontaine, \& Crahay, soumis ; Windschitl, 2002 ; Woolfolk Hoy et al., 2006 ; Yildirim, 2003). Ces contradictions seraient une des caractéristiques les plus importantes des cognitions basées sur l'expérience et ne dérangeraient aucunement l'agir professionnel des enseignants (Leuchter, 2009). Pour cette dernière, les cognitions d'expérience peuvent être centrales ou périphériques. Rejoignant Rokeach (1976) et Richardson (1996), elle considère que les cognitions centrales d'expérience, tels que les préjugés, seraient difficilement changeables, voire impossibles à faire évoluer, car la remise en question de ce type de cognition touche l'individu dans le plus profond de son être. Les cognitions périphériques seraient, selon cet auteur, moins douloureuses à faire évoluer.

En définitive, la principale évolution que subissent les croyances et les connaissances des enseignants en cours de carrière procèderait de leurs mises en acte. Pour les praticiens, il s'agirait moins de changer de croyances pour aller vers des idées plus justes, plus validées, que d'aller vers des croyances de plus en plus "encapsulées " dans l'action afin de rendre celle-ci de mieux en mieux adaptée au contexte d'enseignement.

Marcel Crahay Marcel.Crahay@unige.ch Développement, apprentissage et intervention en situations scolaires, Université de Genève, Suisse et Approches psychopédagogiques et apprentissages scolaires, Université de Liège, Belgique

Philippe Wanlin Développement, apprentissage et intervention en situations scolaires, Université de Genève, Suisse

Élisabeth Issaieva Développement, apprentissage et intervention en situations scolaires, Université de Genève, Suisse

Isabelle Laduron Approches psychopédagogiques et apprentissages scolaires, Université de Liège, Belgique 
(1) II y a là un parti pris quelque peu commode qui se justifie toutefois dans la mesure où, par-delà les différences terminologiques, un courant de recherche relatif aux enseignants porte sur leurs " constructions mentales de l'expérience - souvent condensées et intégrées dans des schémas ou des concepts » (Sigel, 1985 p. 351). Pour notre part, nous utiliserons prioritairement le terme " croyance ", mais aussi celui de " conception ", en le considérant comme synonyme de croyance.

(2) Notons cependant que certains auteurs contestent radicalement le caractère essentiellement propositionnel des croyances (cf. notamment Calderhead, 1996). Nous y reviendrons.

(3) Cette propriété permet de distinguer le domaine des teachers' beliefs and knowledge du courant de recherche désigné par l'expression teacher thinking. Les recherches propres à ce courant portent principalement sur le processus de planification des leçons (teacher planning), sur celui de décision ou de modulation de l'action en classe (interactive decision making) ainsi que sur celui de réflexion a posteriori (post-active reflecting). Bien sûr, il y a des recouvrements importants entre ces deux champs de recherche. II va de soi que les enseignants mobilisent des croyances lorsqu'ils planifient une leçon ou prennent une décision interactive, mais la priorité est différente: dans un cas, il porte sur le contenu; dans l'autre, sur le processus.

(4) Dans son Vocabulaire technique et critique de la philosophie, Lalande (2002) signale que le terme " croyance » a, en philosophie, pris un sens particulier depuis Kant, qui propose de l'utiliser "lorsque l'assentiment n'est suffisant qu'au point de vue subjectif et qu'il est tenu pour insuffisant au point de vue objectif » (p. 198). Dès lors, paraphrasant Kant, on pourrait dire que la croyance se caractérise par un assentiment subjectif fort et, dans un certain nombre de cas, un assentiment objectif faible, voire insuffisant.

(5) Cependant, dans la troisième édition du Handbook of research on teacher education, l'association entre les termes "croyances » et "connaissances » disparaît au profit de la notion de " capacity ", qui rassemble ces deux termes en les combinant avec les traits de personnalité des enseignants, leurs attitudes, leurs compétences et leur perceptions quant à leur compétence personnelle (Grant, 2008; Grant \& Agosto, 2008; Howard \& Aleman, 2008; McDiarmid \& Clevenger-Bright, 2008). II ne faut pas comprendre le terme de capacity comme la capacité telle que définie par De Landsheere (1979), mais plutôt comme renvoyant à un contenu cognitif ou à ce que l'on pourrait qualifier de «facultés » ou de «cognitions". De plus, les auteurs actuels insistent sur le fait que la notion de capacity (ou de cognition) est évolutive car elle suggère une certaine possibilité de la part des enseignants à continuer de développer leurs connaissances, croyances et compétences à travers le temps (McDiarmid \& Clevenger-Bright, 2008).

(6) Le texte anglais auquel nous renvoyons est le suivant : "Teacher belief is a particularly provocative form of personal knowledge that is generally defined as pre- or invervice teachers' implicit assumptions about students, learning, classrooms, and the subject matter to be taught. " (Kagan, 1992, p. 66)

(7) Kagan (1992) écrit : "As a domain, teaching is characterized by an almost total absence of truths, unimpeachably "correct" answers to the most important issues: why students behave as they do, the nature of learning and cognitive growth, the best way to structure a lesson, etc. " (p. 73)

(8) Les idées, croyances et concepts qui règlent notre pensée ne sont pas de nature purement intellectuelle. Selon Lakoff (1985) en particulier, ils prennent souvent la forme de métaphore. Dans Les métaphores dans la vie quotidienne, il montre que nous n'avons pas conscience de notre système conceptuel ; celui-ci s'exprime notamment au travers de métaphores, ce qui l'amène à proposer la notion de « métaphore conceptuelle ». L'idée est donc qu'en utilisant ou en choisissant telle métaphore plutôt que telle autre, un sujet dévoile une part du système conceptuel qui structure sa pensée. Partant de là, des chercheurs ont eu recours à cette technique pour étudier les croyances des ensei- gnants en leur soumettant des métaphores (« À la naissance, l'esprit est un écrin vide... » ; " L'enfant est une belle plante qu'il convient de couvrir de soins attentifs ») et en leur demandant lesquelles ils privilégient.

(9) À noter que notre texte prend également en compte les ajustements (mineurs) apportés par divers publications récentes.

(10) Pour notre part, nous restons fidèles à l'expression teachers' beliefs and knowledge, l'expression teachers' capacity ne nous paraissant pas clarificatrice. De plus, la traduction française de " capacité(s) des enseignants " nous paraît particulièrement équivoque. En fait, nourris à la mamelle de la théorie piagétienne, nous serions prêts à nous laisser tenter par le retour du concept de schème.

(11) Le lecteur aura compris que notre analyse nous conduit à considérer ces quatre termes sinon comme synonymes, au moins comme intimement reliés.

(12) Ainsi cet auteur reprend le terme de schizophrenic tasks (Lieberman, 1992) pour désigner les doubles contraintes qui pèsent sur les enseignants: ceux-ci sont tenus de différencier l'enseignement tout en gardant le contrôle sur l'ensemble de la classe, de tenir compte des intérêts individuels tout en atteignant avec tous un socle commun de compétences. Ces tâches «schizophréniques » placent les enseignants en situation de dilemme quasi permanent (Wanlin \& Crahay, soumis).

(13) Rappelons à cet égard la distinction de Rozenholtz (1989) entre écoles à bas consensus et écoles à haut consensus.

(14) Ainsi Kagan (1992) écrit: “We cannot expect any program of inservice teacher education to effect change in teachers' behaviors without also effecting change in their personal beliefs " (p. 77). Ailleurs, dans le même texte, il dit la même chose concernant la preservice teacher education.

(15) Sur cette distinction, voir notamment Cuban (1988).

(16) On trouvera aussi, dans cette ancienne note de synthèse, la distinction entre ces deux niveaux de changement (de surface et en profondeur) et, dans la foulée, une typologie des changements envisageables en ce qui concerne l'enseignement.

(17) Le terme voluntary a une acception qui se situe entre intentionnel et bénévole. Richardson et Placier (2001) désignent par là, en fait, les changements qui ne sont pas impulsés délibérément de l'extérieur comme le sont ceux qui résultent d'un dispositif de formation.

(18) Dans la synthèse de Richardson et Placier (2001), les deux premières catégories relèvent de ce qu'elles nomment «individual and small group changes ».

(19) Originellement, Fuller (1969) distinguait trois stades de développement: "le stade a-pédagogique", "le stade autocentré et centré sur la matière » et «le stade allo-centré sur les élèves ». Dans le texte, nous reprenons ce qui est le plus souvent mentionné dans la littérature subséquente et qui s'inspire de la publication de 1975 (Fuller \& Bown, 1975), la terminologie renvoyant, au moins en partie, aux travaux de Bullough (1987) et de Ryan (1986).

(20) On ne peut exclure, de la part des enseignants, des moments de déprime et des processus de déclin qu'il est moins fréquent d'observer dans le développement des compétences cognitives des enfants.

(21) Teacher education and learning to teach, programme dirigé par le Centre de recherche sur la formation des enseignants de l'Université du Michigan.

(22) Notons que, dans la plupart des études qui concluent à un changement, les auteurs précisent que certains étudiants changent leurs croyances et/ou que la transformation des croyances se produit à des degrés divers selon les étudiants (Richardson, 1996).

(23) Dans l'étude de Crahay et Ory (2006), les formateurs en psychopédagogie des HEP sont convaincus que leurs étudiants ont cette idée ancrée en eux. Par ailleurs, cette étude met en évidence que ces formateurs sous-estiment leur influence sur les étudiants. 
(24) On ne peut non plus exclure un effet des croyances des enseignants en fonction côtoyés à l'occasion des stages.

(25) Ils écrivent: «To conclude, a number of aspects of a normativeeducative staff development process work to enhance learning and change. " (Richardson \& Placier, 2001, p. 921) Notons cependant que nous n'avons pas trouvé de preuve de cette affirmation.

(26) La question n'est pas simple, car il nous semble que, dans certains cas, le chercheur n'a pas le choix. Comment en effet changer les pratiques des enseignants en matière de redoublement sans changer au préalable ou de manière concomitante leurs croyances? En leur imposant ce changement par une sorte de décret ? Mais l'imposition même de cette pratique risque d'induire une hostilité qu'il va falloir vaincre; les expériences menées en Communauté française de Belgique l'attestent (Crahay \& Donnay, 2001). En revanche, il nous semble envisageable de modifier les pratiques des enseignants concernant l'enseignement de la lecture en recourant à des manuels scolaires et ensuite de travailler sur le changement de croyances. Bref, dans le cas de l'enseignement de la lecture, il paraît possible de comparer deux stratégies de changement des pratiques et des croyances, l'une débutant par les pratiques et l'autre par les croyances. À notre connaissance, ce type d'étude n'a pas encore été réalisé.
(27) II est possible ici d'établir un parallélisme avec les travaux de psychologie sociale sur les biais cognitifs. Rappelons notamment ceux de Lord, Ross et Lepper (1979) qui montrent que des étudiants ayant des attitudes opposées peuvent trouver dans un même texte des arguments qui les confortent dans leur position.

(28) Se basant sur l'étude de cas de deux étudiants, ce chercheur montre que ceux-ci ne changent pas leurs croyances à propos de «la bonne façon d'enseigner " suite aux cours donnés en formation. II émet l'hypothèse que, si ces futurs enseignants persistent dans leurs croyances, c'est parce que le processus de formation ne leur offre pas réellement l'opportunité de confronter leurs croyances aux connaissances enseignées.

(29) La citation anglaise est la suivante: "To promote conceptual change among students, teachers must (a) help students make their implicit beliefs explicit; (b) confront students with the inadequacy or inconsistency of those beliefs; and (c) give students extended opportunities to integrate and differentiate the old and the new knowledge, eliminating brittle preconceptions and elaborating anchors. "(Kagan, 1992, p. 76)

(30) Rappelons que les recherches quasi expérimentales montrent qu'au cours de l'année répétée, les redoublants progressent, mais moins que leurs condisciples aussi faibles qui ont été promus (Crahay, 2004).

\section{BIBLIOGRAPHIE}

ABRIC J.-C. (1994). « Les représentations sociales : aspects théoriques ». In J.-C. Abric, Pratiques sociales et représentations. Paris : PUF, p. 11-36.

AMMON P. (1991). "Using case discussions to promote change in beliefs among mathematics teachers. Expertise in teaching from a developmental perspective. The developmental teacher education program at Berkeley ». Paper presented at the Annual meeting of the American educational research association, Chicago.

ANDERS P. \& RICHARDSON V. (1991). «Research directions: Staff development that empowers teachers' reflection and enhances instruction ". Language Arts, vol. $68, n^{\circ} 4$, p. 316-321.

ASHTON P. (1984). «Teacher efficacy: A motivational paradigm for effective teacher education ". Journal of Teacher Education, vol. 35, n 5, p. 28-32.

AVIGDOR R. (1953). «Étude expérimentale de la genèse des stéréotypes ». Cahiers internationaux de sociologie, $\mathrm{n}^{\circ} 14$, p. $154-168$

BALL D. (1988). "Unlearning to teach mathematics ». For the Learning of Mathematics, vol. 8, $\mathrm{n}^{\circ} 1$, p. 40-48.

BALL D. (1989). «Breaking with experience: The role of a preservice methods course ». Communication présentée à l'Annual meeting of the American educational research association, San Francisco. Disponible sur Internet à l'adresse : <http://ncrtl.msu.edu/http/ipapers/html/ pdf/ip8910.pdf> (consulté le 26 août 2010).

BALL S. \& GOODSON I. (1985). «Understanding teachers: concepts and contexts ». In S. Ball \& I. Goodson, Teachers' lives and careers. Londres : Falmer Press, p. 1-26.

BANDURA A. (1986). Social foundations of thought and action: A social cognitive theory. Englewood Cliffs: Prentice-Hall.

BARNETT C. \& SATHER S. (1992). " Using case discussions to promote change in beliefs among mathematics teachers ". Communication présentée à l'Annual meet- ing of the American educational research association, San Francisco.

BAUMERT J. \& KUNTER M. (2006). « Stichwort: Professionelle Kompetenz von Lehrkräften ». Zeitschrift für Erziehungswissenschaft, $\mathrm{n}^{\circ} 4$, p. 469-520.

BERLINER D. (1994). «Expertise: The wonder of exemplary performances ». In J. Mangiere \& C. Block, Creating powerful thinking in teachers and students: Diverse perspectives. Fort Worth : Harcourt Brace College, p. 161-186.

BLACK A. \& AMMON P. (1992). «A developmentalconstructivist approach to teacher education ". Journal of Teacher Education, vol. 43, n 5, p. 323-335.

BLUMENFELD P., KRACJIK J., MARX R. \& SOLOWAY E. (1994). "Lessons learned: How collaboration helped middle-grade science teachers learn project-based instruction ". Elementary School Journal, vol. 94, n 5 , p. 539-551.

BOLIN F. (1990). «Helping student teachers think about teaching: Another look at Lou ». Journal of Teacher Education, vol. 41, $\mathrm{n}^{\circ} 1$, p. 10-19.

BOOK C. \& FREEMAN D. (1986). « Differences in entry characteristics of elementary and secondary teacher candidates ". Journal of Teacher Education, vol. 37, $\mathrm{n}^{\circ} 2$, p. 47-51.

BOOK C., BYERS J. \& FREEMAN D. (1983). «Student expectations and teacher education traditions with which we can and cannot live ". Journal of Teacher Education, vol. 34, $\mathrm{n}^{\circ} 1$, p. 1-13.

BORKO H. \& PUTNAM R. (1996). «Learning to teach". In D. Berliner \& R. Calfee, Handbook of educational psychology. New York: MacMillan, p. 673-708.

BORKO H. \& SHAVELSON R. (1990). « Teacher decision making ». In B. Jones \& L. Idol, Dimensions of thinking and cognitive instruction. New Jersey : Erlbaum, p. 311-346.

BOS C. \& ANDERS P. (1994). "The study of student change ". In V. Richardson, Teacher change and the 
staff development process. New York: Teachers College Press, p. 181-198.

BROMME R. (1992). Des Lehrer als Experte: Zur Psychologie des professionellen Wissens. Berne : Hans Huber.

BRONFENBRENNER U. (1986). "Ecology of the family as a context for human development: Research perspectives ». Developmental Psychology, vol. 22, n 6, p. 723-742.

BROOKHART S. \& FREEMAN D. (1992). " Characteristics of entering teacher candidates ". Review of Educational Research, vol. 62, $\mathrm{n}^{\circ}$ 1, p. 37-60.

BROUDY H. (1980). "What do professors of education profess? » The Educational Forum, vol. 44, n 4, p. 441-451.

BUCHMANN M. (1986). "Role over person: legitimacy and authenticity in teaching ". In M. Ben Peretz, R. Bromme \& R. Halkes, Advances of research on teacher thinking. Lisse : Swets \& Zeitlinger B.V., p. 55-69.

BUCHMANN M. (1987). "Role over person: Justifying teacher action and decisions ". Scandinavian Journal of Educational Research, vol. 31, n 1, p. 1-21.

BULLOUGH R. (1987). «First-year teaching: A case study ». Teachers College Record, vol. 89, n², p. 219-237.

BULLOUGH R. \& BAUGHMAN K. (1997). First-year teacher. Eight years later: An inquiry into teacher development. New York: Teachers College Press.

BULLOUGH R. \& KNOWLES J. (1990). « Becoming a teacher: Struggles of a second-career beginning teacher ». International Journal for Qualitative Studies in Education, vol. $3, n^{\circ} 2$, p. 101-112.

BULLOUGH R. \& KNOWLES J. (1991). « Teaching and nurturing: Changing conceptions of self as teacher in a case study of becoming a teacher ». Qualitative Studies in Education, vol. 4, $\mathrm{n}^{\circ}$ 2, p. 121-140.

BUTT R., RAYMOND D., McCUE G. \& YAMAGISI L. (1992). "Collaborative autobiography and the teacher's voice". In I. Goodson, Studying teachers' lives. New York: Teachers College Press, p. 51-98.

CALDERHEAD J. (1988). "The contribution of field experiences to student primary teachers' professional learning ". Research in Education, $n^{\circ} 40$, p. 34-49.

CALDERHEAD J. (1996). "Teachers: Beliefs and knowledge ". In D. Berliner \& R. Calfee, Handbook of educational psychology. New York : MacMillan, p. 673-708.

CALDERHEAD J. \& ROBSON M. (1991). «Images of teaching: Student teachers' early conceptions of classroom practice ". Teaching \& Teacher Education, vol. $7, \mathrm{n}^{\circ} 1$ p. 1-8.

CARPENTER T., FENNEMA E., PETERSON P., CHIANG C. \& LOEF M. (1989). "Using knowledge of children's mathematics thinking in classroom teaching: An experimental study ». American Educational Research Journal, vol. $26, n^{\circ} 4$, p. 499-531.

CHIN R. \& BENNE K. (1969). "General strategies for effecting changes in human systems ». In W. Bennis, K. Benne \& R. Chin, The planning of change $\left[2^{\mathrm{e}}\right.$ éd.]. New York: Holt, Rinehart \& Winston, p. 32-59.

CIVIL M. (1993). " Prospective elementary teachers' thinking about teaching mathematics ». Journal of Mathematical Behavior, vol. 12, $\mathrm{n}^{\circ} 1$, p. 79-109.

CIVIL M. (1995). “ Listening to students' ideas: Teachers interviewing in mathematics ». In L. Meira \& D. Caraher, Proceedings of the 19th Psychology of mathematics education conference, vol. 2. Recife, Brésil : Universidade Federal do Pernambuco, p. 154-161.
CIVIL M. (1996). "Thinking about mathematics and its teaching: An experience with preservice teachers". In J. Gimenez, S. Llinares \& V. Sanchez, Becoming a primary teacher: Issues from mathematics education. Badajoz : Autores, p. 137-154.

CLARK C. \& PETERSON P. (1986). «Teachers' thought processes ". In M. Wittrock, Handbook of research on teaching [ $3^{e}$ éd.]. New York : MacMillan, p. 255-296.

COCHRAN-SMITH M. (1991). "Learning to teach against the grain ». Harvard Educational Review, vol. 61, $\mathrm{n}^{\circ} 3$, p. 279-310.

COHEN D. (1990). «A revolution in one classroom: The case of Mrs Oublier». Educational Evaluation and Policy Analysis, vol. 12, $\mathrm{n}^{\circ} 3$, p. 311-344.

COMEAUX M. (1992). «Challenging students' views about teaching and learning: Constructivism in social foundations classroom ". Communication présentée à l'Annual meeting of the American educational research association, San Francisco.

CONNERS R. (1978). An analysis of teacher thought processes, beliefs, and principles during instruction. Thèse de doctorat non publiée, sciences de l'éducation, University of Alberta, Edmonton, Canada.

CORNETT J. (1990). «Teacher thinking about curriculum and instruction: A case study of a secondary social studies teacher". Theory and Research in Social Education, vol. 18, $\mathrm{n}^{\circ} 3$, p. 248-273.

CRAHAY M. (1989). «Contraintes de situation et interactions maître-élève: changer sa façon d'enseigner, est-ce possible ? "Revue française de pédagogie, $\mathrm{n}^{\circ} 88$, p. 67-94.

CRAHAY M. (2000). L'école peut-elle être juste et efficace? De l'égalité des chances à l'égalité des acquis. Bruxelles: De Boeck.

CRAHAY M. (2004). «Peut-on conclure à propos des effets du redoublement ? ". Revue française de pédagogie, $\mathrm{n}^{\circ} 148$, p. 11-23.

CRAHAY M. (2006). Peut-on lutter contre l'échec scolaire ? [3 $3^{e}$ éd.]. Bruxelles : De Boeck.

CRAHAY M. \& DONNAY J. (2001). La mise en place de la réforme du premier degré de l'enseignement secondaire. Recherche interuniversitaire, Rapport de recherche auprès du ministre de l'Enseignement secondaire, Université de Liège.

CRAHAY M. \& FAGNANT A. (2009). «À propos de l'épistémologie personnelle: un état des recherches anglosaxonnes ". Revue française de pédagogie, $\mathrm{n}^{\circ} 161$, p. 79-117.

CRAHAY M. \& MONSEUR C. (2006). « Différences individuelles et effets d'agrégation en ce qui concerne les performances en lecture. Analyse secondaire des données PISA 2000 ». In C. Houssemand, R. Martin \& P. Dickes, Perspectives de psychologie différentielle. Rennes: Presses universitaires de Rennes, p. 23-34.

CRAHAY M. \& ORY P. (2006). "Les représentations des normaliens en matière de redoublement évoluent-elles au cours de leur formation à l'école normale ?" Communication présentée au $4^{\mathrm{e}}$ congrès des chercheurs en éducation, Bruxelles.

CRONIN-JONES L. (1991). «Science teacher beliefs and their influence on curriculum implementation: Two case studies ". Journal of Research in Science Teaching, vol. $28, n^{\circ} 3$, p. $235-250$. 
CUBAN L. (1984). How Teachers Taught: Constancy and Change in American Classrooms, 1890-1980. New York : Longman.

CUBAN L. (1988). « Constancy and change in schools (1880s to the present) ". In P. Jackson, Contributing to educational change: Perspectives on research and practice. Berkeley : McCutcheon, p. 85-106.

DE LANDSHEERE G. (1979). Dictionnaire de l'évaluation et de la recherche en éducation. Paris : PUF.

DEWEY J. (1933). How we think: A restatement of the relation of reflective thinking to the educative process. Boston : D. C. Heath.

DIEDRICH M., THUSSBAS C. \& KLIEME E. (2002). «Professionnelles Lehrerwissen uns selbstberichte Unterrichtspraxis im Fach Mathematik ». Zeitschrift für Pädagogik, $\mathrm{n}^{\circ} 45$ [Beiheft], p. 107-123.

DOISE W. (1973). "Relations et representations intergroupes ». In S. Moscovici, Introduction à la psychologie sociale, vol. 2. Paris : Larousse.

DOUDIN P.-A., PFULG L., MARTIN D. \& MOREAU J. (2001). «Entre renoncement et engagement. Un défi pour la formation continuée des enseignants ». In L. Lafortune, C. Deaudelin, P.-A. Doudin \& D. Martin, La formation continue. De la réflexion à l'action. Québec: Presses de I'Université du Québec.

DOYLE W. (1975). "Paradigms for research on teacher effectiveness ". Communication présentée à l'Annual meeting of the American educational research association, Washington.

DOYLE W. (1977). "Paradigms for research on teacher effectiveness ». Review of Research in Education, vol. 5, $\mathrm{n}^{\circ} 1$, p. 163-198.

DOYLE W. (1979). «Making managerial decisions in classrooms ". In D. Duke, Classroom management: The 78th yearbook of the National society for the study of education. Chicago : University of Chicago Press, p. 42-74.

DOYLE W. (1986). «Classroom organisation and management ». In M. Wittrock, Handbook of research on teaching [ $3^{e}$ éd.]. New York: MacMillan, p. 392-431.

DOYLE W. \& PONDER G. (1977). « The practicality ethic in teacher decision-making ». Interchange, vol. $8, \mathrm{n}^{\circ} 3$, p. 1-12.

DRAELANTS H. (2006). « Le redoublement est moins un problème qu'une solution: comprendre l'attachement social au redoublement en Belgique francophone ". Les cahiers de recherche en éducation et formation, $n^{\circ} 52$, p. 3-21.

DRAELANTS H. (2009). Réforme pédagogique et légitimation. Le cas d'une politique de lutte contre le redoublement. Bruxelles : De Boeck.

DUFFY G. \& ROEHLER L. (1986). «Constraints on teacher change ». Journal of Teacher Education, vol. 35, p. 55-58.

EVERTSON C. \& WEINSTEIN C. (2006). "Classroom management as a field of inquiry ». In C. Evertson \& C. Weinstein, Handbook of classroom management: Research, practice, and contemporary issues. Mahwah : Lawrence Erlbaum, p. 3-16.

FEIMAN-NEMSER S. (1983). «Learning to teach ». In L. Shulman \& G. Sykes, Handbook of teaching and policy. New York : Longman, p. 150-171.
FEIMAN-NEMSER S. (1990). «Teacher preparation: Structural and conceptual alternatives". In R. Houston, Handbook of research on teacher education. New York: McMillan, p. 212-233.

FEIMAN-NEMSER S. \& BUCHMANN M. (1989). « Describing teacher education: A framework and illustrative findings from a longitudinal study of six students ". The Elementary School Journal, vol. 89, $n^{\circ} 3$, p. 365-377.

FEIMAN-NEMSER S., McDIARMID G., MELNICK S. \& PARKER M. (1989). Changing beginning teachers' conceptions: A description of an introductory teacher education course. East Lansing: National center for research on teacher education, College of education, Michigan State University.

FENNEMA E., CARPENTER T., FRANKE M. \& CAREY D. (1992). « Learning to use children's mathematical thinking: A case study ». In R. Davis \& C. Maher, Schools, mathematics and the world of reality. Needham Heights : Allyn \& Bacon, p. 93-117.

FENSTERMACHER G. (1979). «A philosophical consideration of recent research on teacher effectiveness". Review of research in education, vol. 6, $\mathrm{n}^{\circ} 1, \mathrm{p} .157-185$.

FENSTERMACHER G. (1983). « How should implications of research on teaching be used? "Elementary School Journal, vol. 83, $n^{\circ} 4$, p. 496-499.

FENSTERMACHER G. (1984a). "On getting from here (research) to there (practice)». In R. Egbert \& M. Kluender, Using research to improve teacher education. Washington : Clearinghouse, p. 22-27.

FENSTERMACHER G. (1984b). «What counts as research on teacher education? » In S. Hord, S. O'Neal \& M. Smith, Beyond the looking glass: Papers from a national symposium on teacher education policies, practices \& research. Austin: University of Texas at Austin, p. 27-33.

FENSTERMACHER G. (1985). "Who decides the worth of educational research? » In E. Robertson, Philosophy of education, 1984. Proceedings of the fortieth Annual meeting of the philosophy of education society. Normal : Philosophy of Education Society, p. 295-298.

FENSTERMACHER G. (1986). "Philosophy of research on teaching: Three aspects ". In M. Wittrock, Handbook of research on teaching [ $3^{\mathrm{e}}$ éd.]. New York: MacMillan, p. 37-49.

FENSTERMACHER G. (1994). "The knower and the known: The nature of knowledge in research on teaching". Review of research in education, vol. 20, $\mathrm{n}^{\circ} 1$, p. 3-56.

FLODEN R. \& CLARK C. (1988). "Preparing teachers for uncertainty ". Teacher College Record, vol. 89, $n^{\circ} 4$, p. 505-524.

FREEMAN D. (1991). « To make the tacite explicite: Teacher education, emerging discourse, and conceptions of teaching ». Teaching and Teacher Education, vol. 9, $n^{\circ} 5-6$, p. 439-454.

FREEMAN D. (1993). «Renaming experience/reconstructing practice: Developing new understandings of teaching ". Teaching and Teacher Education, vol. 9, $\mathrm{n}^{\circ}$ 5-6, p. $485-497$.

FULLAN M. (1990). "Staff development, innovation and institutional development». In B. Joyce, Changing school culture through staff development. Alexandria: Association for supervision and curriculum development, p. 3-25. 
FULLAN M. (1991). The new meaning of educational change [2 $2^{\mathrm{e}}$ é.]. New York : Teachers College Press.

FULLER F. (1969). "Concerns of teachers: A developmental conceptualization". American Educational Research Journal, vol. $6, \mathrm{n}^{\circ} 2$, p. 207-226.

FULLER F. \& BOWN O. (1975). «Becoming a teacher». In K. Ryan, The 74th yearbook of the National society for the study of education, part 2. Chicago : University of Chicago Press, p. 25-52.

GAGE N.-G. (1986). «Comment tirer un meilleur parti des recherches sur les processus d'enseignement». In M. Crahay \& D. Lafontaine, L'art et la science de l'enseignement. Bruxelles : Labor.

GASTAGER A. (2003). Paradigmenvielfalt aus Sicht des Unterrichtenden. Lengerich : Pabst.

GRANT C. (2008). "Teacher capacity ». In M. CochranSmith, S. Feiman-Nemser, D. Mclntyre \& K. Demers, Handbook of research on teacher education [ $3^{\mathrm{e}}$ éd.]. New York : Routledge, p. 127-133.

GRANT C. \& AGOSTO V. (2008). "Teacher capacity and social justice in teacher education ». In M. CochranSmith, S. Feiman-Nemser, D. Mclntyre \& K. Demers, Handbook of research on teacher education [3 $3^{\mathrm{e}}$ éd.]. New York : Routledge, p. 175-200.

GREEN T. (1971). The activities of teaching. New York: McGraw-Hill.

GRUBER H. (1999). Erfahrung als Grundlage kompetenten Handelns. Berne : Huber.

GURNEY B. (1995). "Tugboats and tennis games: Preservice conception of teaching and learning revealed through metaphors ". Journal of Research in Science Teaching, vol. 32, $n^{\circ}$ 6, p. 569-583.

GUSKEY T. (1986). "Staff development and the process of teacher change ». Educational Researcher, vol. 15, $\mathrm{n}^{\circ} 5$, p. 5-12

HALKES R. \& DEIJKERS R. (2003). «Teachers' teaching criteria ». In M. Kompf \& P. Denicolo, Teacher thinking twenty years on: Revisiting persisting problems and advances in education. Lisse : Swets \& Zeitlinger B.V., p. 3-13.

HANCOCK E. \& GALLARD A. (2004). «Preservice science teachers' beliefs about teaching and learning: The influence of K-12 field experiences ". Journal of Science Teacher Education, vol. 15, n 4, p. 281-291.

HARINGTON H. \& HATHAWAY R. (1994). "Computer conferencing, critical reflection, and teacher development ». Teaching and Teacher Education, vol. 10, $\mathrm{n}^{\circ} 2$, p. 543554.

HARITOS C. (2004). «Understanding teaching through the minds of teacher candidates: A curious blend of realism and idealism ". Teaching and Teacher Education, vol. $20, n^{\circ} 6$, p. 637-654.

HARVEY O. (1986). "Belief systems and attitudes toward the death penalty and other punishments ". Journal of Personality, vol. 54, $\mathrm{n}^{\circ}$ 4, p. 659-675.

HAUSOUL E. (2005). Quels sont les effets de l'insertion professionnelle des enseignants sur leurs représentations sociales et, plus précisément, sur leur représentation de l'échec scolaire ? Mémoire de licence en sciences de l'éducation non publié, Université de Liège, Liège.

HOBAN G. (1995). «Reflection on what? Assisting teachers to frame and reframe their practice ". Communication présentée à l'Annual meeting of the American educational research association, San Francisco.
HOFER B. \& PINTRICH P. (1997). « The development of epistemological theories: Beliefs about knowledge and knowing and their relation to learning ". Review of Educational Research, vol. 67, $\mathrm{n}^{\circ} 1, \mathrm{p} .88-140$.

HOLLINGSWORTH S. (1989). "Prior beliefs and cognitive change in learning to teach ». American Educational Research Journal, vol. 26, n² 2, p. 160-189.

HOLT-REYNOLDS D. (1992). «Personal history-based beliefs as relevant prior knowledge in course work". American Educational Research Journal, vol. 29, $\mathrm{n}^{\circ} 2$, p. 325349.

HOWARD T. \& ALEMAN G. (2008). «Teacher capacity for diverse learners. What do teacher need to know? » In M. Cochran-Smith, S. Feiman-Nemser, D. McIntyre \& K. Demers, Handbook of research on teacher education [3 ${ }^{e}$ éd.]. New York : Routledge, p. 157-174.

HOY W. (1967). "Organizational socialization: The student teacher and pupil control ideology ". The Journal of Educational Research, vol. 61, n 4, p. 153-259.

HOY W. \& WOOLFOLK A. (1990). "Socialization of student teachers". American Educational Research Journal, vol. $27, n^{\circ} 2$, p. $279-300$.

HUBER G. \& ROTH J. (1990). «Teachers' classroom activities and certainty and incertainty orientation ». In A. Day, Insight into teachers' thinking and action. Londres: Falmer Press, p. 119-132.

HUBERMAN M. (1989). «On teachers' careers: Once over lightly with a broad brush ». International Journal of Educational Research, vol. 13, n 4, p. 347-361.

HUBERMAN A., GROWNVER M. \& MARTIN J. (1989). La vie des enseignants. Neuchâtel : Delachaux et Niestlé. [Traduction anglaise en 1993: The lives of teachers. Londres: Cassell].

ISSAIEVA E. \& CRAHAY M. (2010). «Conceptions de l'évaluation scolaire des élèves et des enseignants : validation d'échelles et étude de leurs relations ". Mesure et évaluation en éducation, vol. $33, \mathrm{n}^{\circ} 1$, p. 31-62.

JENSEN J. (1998). “Teacher candidates' conceptions of teaching and learning: A review ". Communication présentée à l'Annual meeting of the American educational research association, San Diego.

JONES M. \& VESILIND E. (1996). «Putting practice into theory: Changes in the organization of preservice teachers' pedagogical knowledge ". American Educational Research Journal, vol. 33, $\mathrm{n}^{\circ} 1$, p. 91-117.

KAGAN D. (1992). "Implications of research on teacher beliefs ». Educational Psychologist, vol. 27, $\mathrm{n}^{\circ} 1$, p. 65-90.

KIRSCHNER P., SWELLER J. \& CLARK R. (2006). «Why minimal guidance during instruction does not work: An analyis of the failure of constructivist, discovery, problembased, experiential, and inquiry-based teaching ». Educational Psychologist, vol. 41, n² 2, p. 75-86.

KLIEME E. \& REUSSER K. (2003). « Unterrichtsqualität und mathematisches Verständnis im internationalen Vergleich? Ein Forschungsprojekt und erste Schritte zur Realisierung ". Unterrichtswissenschaft, vol. 31, $n^{\circ} 3$, p. 194-205.

KNOWLES J. (1992). « Models for teachers' biographies ». In I. Goodson, Studying teachers' lives. New York: Teachers College Press, p. 99-152. 
KORTHAGEN F. (1988). "The influence of learning orientations on the development of reflective teaching ". In J. Calderhead, Teachers' Professional Learning. Philadelphia : Falmer Press, p. 35-50.

KRAJCIK J., LAYMAN J., STARR M. \& MAGNUSSON S. (1991). "The development of middle school teachers' content knowledge and pedagogical content knowledge of heat energy and temperature ". Communication présentée à l'Annual meeting of the American educational research association, Chicago.

KREMER L. (1981). "Personal characteristics of teachers, situational variables and deliberations in the process of planning instruction ». Research in Education, $\mathrm{n}^{\circ} 26$, p. 21-30.

LAKOFF G. \& JOHNSON M. (1985). Les métaphores dans la vie quotidienne [Trad. de M. de Fornel et J.-J. Lecercle]. Paris : Éd. de Minuit.

LALANDE A. (2002). Vocabulaire technique et critique de la philosophie. Paris : PUF.

LAMPERT M. (1986). «Teachers' strategies for understanding and managing classroom dilemmas". In M. Ben Peretz, R. Bromme \& R. Halkes, Advances of research on teacher thinking. Lisse : Swets \& Zeitlinger B.V., p. 70-83.

LANIER J. \& LITTLE J. (1986). «Research on teacher education ". In M. Wittrock, Handbook of research on teaching [3 $3^{e}$ éd.]. New York: MacMillan, p. 527-569.

LEAVY A., McSORLEY F. \& BOTÉ L. (2007). «An examination of what metaphor construction reveals about the evolution of preservice teachers' beliefs about teaching and learning ". Teaching and Teacher Education, vol. 23 , $\mathrm{n}^{\circ} 7$, p. 1217-1233.

LESTER N. \& ONORE C. (1990). Learning change. Portsmouth : Boynton/Cook.

LEUCHTER M. (2009). Die Rolle der Lehrperson bei der Aufgabenbearbeeitung: Unterrichtsbezogene Kognitionen von Lehrpersonen. Münster : Waxmann.

LEUCHTER M., REUSSER K., PAULI C. \& KLIEME E. (2008). "Zusammenhänge zwischen unterrichtsbezogenen Kognitionen und Handlungen von Lehrpersonen ". In M. Gläser-Zikuda \& J. Seifried, Lehrerexpertise. Analyse und Bedeutung unterrichtlichen Handelns. Münster : Waxmann, p. 165-186.

LEVIN B. \& AMMON P. (1992). "The development of the beginning teachers' pedagogical thinking: A longitudinal analysis of four case studies ". Teacher Education Quarterly, vol. 19, $\mathrm{n}^{\circ} 4$, p. 19-37.

LIEBERMAN A. (1992). «Introduction: The chanching contexts of teaching ». In A. Lieberman, The changing contexts of teaching. Ninety first yearbook of the National society for the study of education. Chicago : University of Chicago Press, p. 1-10.

LINDBLAD S. (1990). "From technology to craft: On teachers' experimental adoption of technology as a new subject in the Swedish primary school ". Journal of Curriculum Studies, vol. 22, $\mathrm{n}^{\circ}$ 2, p. 165-175.

LIPOWSKY F. (2006). « Auf den Lehrer kommt es an. Empirische Evidenzen für Zusammenhänge zwischen Lehrerkompetenzen, Lehrerhandeln und dem Lernen der Schüler ». Zeitschrift für Pädagogik, $\mathrm{n}^{\circ} 51$ [Beiheft], p. 47-70.

LISTON D. \& ZEICHNER K. (1989). «Action research and reflective teaching in preservice teacher education". Communication présentée à l'Annual meeting of the
American educational research association, San Francisco.

LORD C., ROSS L. \& LEPPER M. (1979). « Biased assimilation and attitude polarization: the effects of prior theories on subsequently considered evidence ». Journal of Personality and Social Psychology, vol. 37, $\mathrm{n}^{\circ}$ 11, p. 2098-2109.

LORTIE D. (1975). Schoolteacher. Chicago: University of Chicago Press.

LOUDEN W. (1991). Understanding teaching: Continuity and change in teacher's knowledge. New York: Teachers College Press.

MAHLIOS M. \& MAXSON M. (1998). « Metaphors as structures for elementary and secondary preservice teachers' thinking. " International Journal of Educational Research, vol. 29, $\mathrm{n}^{\circ} 3$, p. 227-240.

MARCOUX G. \& CRAHAY M. (2008). « Mais pourquoi continuent-ils à faire redoubler ? Essai de compréhension du jugement des enseignants". Revue des sciences de l'éducation, vol. 30, $\mathrm{n}^{\circ} 3$, p. 501-518.

MARKES S. (1995). Acquiring pratical knowledge: A study of development through observations of student teaching practice and dialogues of community. Thèse de doctorat non publiée, sciences de l'éducation, University of Arizona, Tucson.

MARKS S. \& GERSTEN R. (1998). «Engagement and disengagement between special and general educators: An application of Miles and Huberman's cross-case analysis ". Learning Disabilities Quarterly, vol. 21, $\mathrm{n}^{\circ} 1$, p. 34-56.

MARLAND P. (1977). A study of teachers' interactive thoughts. Thèse de doctorat non publiée, sciences de l'éducation, University of Alberta, Edmonton, Canada.

MARLAND P. (1993). «Teachers' knowledge of students: A significant domain of practical knowledge? » Disponible sur Internet à l'adresse: <http://www.aare.edu. au/93pap/marlp93147.txt> (consulté le 26 août 2010).

MARTINET C. \& RIEBEN L. (2010). "L'apprentissage initial de la lecture et ses difficultés ». In M. Crahay \& M. Dutrévis, Psychologie des apprentissages scolaires. Bruxelles : De Boeck, p. 189-224.

MAYER D. \& MARLAND P. (1997). « Teachers' knowledge of students: A significant domain of practical knowledge? " Asia-Pacific Journal of Teacher Education, vol. $25, n^{\circ} 1$, p. 17-34.

McDIARMID G. (1990). “Challenging prospective teachers' beliefs in an early field experience: A quixotic undertaking? » Journal of Teacher Education, vol. 29, $\mathrm{n}^{\circ} 1$, p. 21-29.

McDIARMID G. (1992). «What to do about differences? A study of multicultural education for teacher trainees in the Los Angeles unified school district". Journal of Teacher Education, vol. 43, $\mathrm{n}^{\circ} 2$, p. 83-93.

McDIARMID G. \& CLEVENGER-BRIGHT M. (2008). « Rethinking teacher capacity ». In $M$. Cochran-Smith, S. Feiman-Nemser, D. Mclntyre \& K. Demers, Handbook of research on teacher education [ $3^{e}$ éd.]. New York: Routledge, p. 134-156.

McDONALD J. (1986). "Raising the teacher's voice and the ironic role of theory ". Harvard Educational Review, vol. $56, n^{\circ} 4$, p. 355-378.

McLAUGHLIN M. (1994). "Learning from experience: Lessons from policy implementation ". Educational Evaluation and Policy Analysis, vol. 9, n² 2 p. 171-178. 
MELLADO V. (1998). "The classroom practice of preservice teachers and their conceptions of teaching and learning science ". Science Education, vol. 82, n², p. 197-214.

MEVARECH Z. (1995). "Teachers' paths on the way to and from the professional development forum ». In T. Guskey \& M. Huberman, Professional development in education: New paradigms and practices. New York: Teachers College Press, p. 151-170.

MONSEUR C. \& CRAHAY M. (2008). «Composition académique et sociale des établissements, efficacité et inégalités scolaires: une comparaison internationale ». Revue française de pédagogie, $\mathrm{n}^{\circ} 162$, p. 55-65.

MONTGOMERY C., LEGAULT F., GAUTHIER C. \& BUJOLD N. (1999). "Conceptions of an efficient teacher, motivations of career choice, and feelings of efficacy in secondary school student teachers ". Res Academica, vol. 17, $\mathrm{n}^{\circ} 1$, p. 139-163.

MUGNY G. \& CARUGATI F. (1985). « L'intelligence au pluriel. Les représentations sociales de l'intelligence et de son développement ». Cousset : Delval.

MUNBY H. (1982). "The place of the teachers' beliefs in research on teacher thinking and decision making and alternative methodology ». Instructional Science, vol. $11, n^{\circ} 3$, p. 201-225.

MUNBY H. (1983). " A qualitative study of teachers' beliefs and principles". Communication présentée à l'Annual meeting of the American educational research association, Montréal, Canada.

MUNBY H. \& RUSSELL T. (1992). «Transforming chemistry research into chemistry teaching: The complexities of adopting new frames for experience ". In T. Russell \& H. Munby, Teachers and teaching: From classroom to reflection. Londres : Falmer Press, p. 90-123.

MUNBY H. \& RUSSELL T. (1994). «The authority of experience in learning to teach: Messages from a physics methods class ». Journal of Teacher Education, vol. 45, $n^{\circ} 2$, p. 86-95.

MUNBY H., CUNNINGHAM M. \& CHIN P. (1998). "Cooperative education: The functions of experience in workplace learning». Communication présentée à l'Annual meeting of the Canadian society for studies in education, Ottawa, Canada.

MUNBY H., RUSSELL T. \& MARTIN A. (2001). "Teachers' knowledge and how it develops ». In V. Richardson, Handbook of research on technopole. Washington: American educational research association, p. 877904.

MUNRO R. (1993). "A case study of school-based training systems in New Zealand secondary schools". In J. Elliott, Reconstructing Teacher Education. Londres : Falmer Press, p. 95-109.

NAULT T. (1999). L'enseignant et la gestion de la classe. Montréal : Éd. Logiques.

NESPOR J. (1987). "The role of beliefs in the practice of teaching ». Journal of Curriculum Studies, vol. $19, \mathrm{n}^{\circ} 4$ p. 317-328.

NIAS J. (1989). "Teaching and the self». In M. Holly \& C. McLoughlin, Perspectives on teacher professional development. Londres : Falmer press, p. 155-172.

NISBETT R. \& ROSS L. (1980). Human inference: Strategies and shortcomings of social judgment. Englewood Cliffs : Prentice-Hall.
OLSON J. (1980). "Teacher constructs and curriculum change ". Journal of Curriculum Studies, vol. $1, \mathrm{n}^{\circ} 1$, p. 1-11.

OLSON J. (1981). "Teacher influence in the classroom. A context for understanding curriculum translation ». Instructional Science, vol. 10, n 3, p. 259-275.

OLSON M. (1993). "Knowing what counts in teacher education ». Communication présentée à la Canadian association of teacher educators, Canadian society of studies in education, Ottawa, Canada.

OPDENAKKER M. \& VAN DAMME J. (2006). "Teacher characteristics and teaching styles as effectiveness enhancing factors of classroom pratice ». Teaching and Teacher Education, vol. 22, n¹, p. 1-21.

PAJARES F. (1992). "Teachers' beliefs and educational research: Cleaning up a messy construct ". Review of Educational Research, vol. 62, n 3, p. 307-332.

PALINSCAR A., MAGNUSSON S., MARANO N., FORD D. \& BROWN N. (1998). "Designing a community of practice: Principles and practices of the GIsML community ". Teaching and Teacher Education, vol. 14, n 1, p. 5-19.

PETERSON P., FENNEMA E., CARPENTER T. \& LOEF M. (1989). «Teachers' pedagogical content beliefs in mathematics ". Cognition and Instruction, vol. 6, $\mathrm{n}^{\circ} 1$, p. 1-40.

PHILIP R., ARMSTRONG B. \& BEZUK N. (1993). «A preservice teacher learning to teach mathematics in a cognitively guided manner». In J. Becker \& B. Pence, Proceedings of the fifteenth annual meeting of the PMENA, vol. 2. Pacific Grove: San Jose State University, p. $159-165$

PIAGET J. (1975). L'équilibration des structures cognitives: problème central du développement. Paris : PUF.

PINES A. \& WEST L. (1986). "Conceptual understanding and science learning: An interpretation of research within a sources-of-knowledge framework". Science Education, vol. $70, \mathrm{n}^{\circ}$ 5, p. 583-604.

PLACIER P. \& HAMILTON M. (1994). « Schools as contexts: A complex relationship ". In V. Richardson, Teacher change and the staff development process: A case in reading instruction. New York : Teachers College Press, p. $135-159$

PRAWATT R. (1992). "Are changes in views about mathematics teaching sufficient? The case of a fifth-grade teacher ". The Elementary School Journal, vol. 93, $n^{\circ} 2$, p. 195-211.

REMILLARD J. (2005). " Examining key concepts in research on teachers' use of mathematics curricula ». Review of Educational Research, vol. 75, n² 2, p. 211-246.

RICHARDSON V. (1990). "Significant and worthwhile change in teaching practice ». Educational Researcher, vol. 19, $\mathrm{n}^{\circ} 7$, p. $10-18$

RICHARDSON V. (1994). "The consideration of beliefs in staff development ». In V. Richardson, Teacher change and the staff development process: A case of reading instruction. New York: Teachers College Press, p. 90-108.

RICHARDSON V. (1996). "The role of attitudes and beliefs in learning to teach ». In J. Sikula, Handbook of Research on Teacher Education. New York: MacMillan, p. 102-119.

RICHARDSON V. (1998). "Professional development in the instruction of reading ". In J. Osborn \& F. Lehr, Literacy for all: Issues in teaching and learning. Chicago: Guilford Press, p. 303-318. 
RICHARDSON V. \& HAMILTON M. (1994). "The practical argument staff development process ». In V. Richardson, Teacher change and staff development process: A case in reading instruction. New York: Teachers College Press, p. 109-134.

RICHARDSON V. \& PLACIER P. (2001). «Teacher change ». In V. Richardson, Handbook of research on teaching. Washington: American educational research association, p. 905-947.

RICHARDSON V., ANDERS P., TIDWELL D. \& LLOYD C (1991). "The relationship between teachers' beliefs and practices in reading comprehension instruction ". American Educational Research Journal, vol. 28, $\mathrm{n}^{\circ} 3$, p. 559-586.

RICHARDSON-KOEHLER V. (1988). «Barriers to the effective supervision of student teaching: A field study ". Journal of Teacher Education, vol. 39, $\mathrm{n}^{\circ} 2$, p. 28-34.

ROKEACH M. (1976). Beliefs, attitudes and values: A theory of organization and change. San Francisco : Jossey-Bass.

ROSS D., JOHNSON M. \& SMITH W. (1991). « Developing a professional teacher at the University of Florida ». Communication présentée à l'Annual meeting of the American educational research association, Chicago.

ROZENHOLTZ (1989). Teachers' workplace: The social organization of schools. New York : Longman.

RUMPF H. \& KRANICH E. (2000). Welche Art von Wissen braucht des Lehrer? Ein Einspruch gegen landläufige Praxis. Stuttgart : Klett.

RUSSELL T. (1988). «From pre-service teacher education to first year of teaching: A study of theory and practice ». In J. Calderhead, Teachers' professional learning. Londres : Falmer Press, p. 13-34.

RUSSELL T. (1995). "A teacher educator and his students reflect on teaching high school physics ". Teacher Education Quarterly, vol. 22, $n^{\circ} 3$, p. 85-98.

RUSSELL T., MUNBY H., SPAFFORD C. \& JONSTHON P. (1988). " Learning the professional knowledge of teaching ». In P. Grimment \& G. Erickson, Reflection in teacher education. New York: Teachers College Press, p. $67-90$

RYAN K. (1986). « Lives of first year teachers ». Communication présentée à l'Annual meeting of the American association of teacher educators, Washington.

SABAN A., KOCBEKER B. \& SABAN A. (2007). "Prospective teachers' conceptions of teaching and learning revealed through metaphor analysis ". Learning and Instruction, vol. $17, \mathrm{n}^{\circ} 2$, p. 123-139.

SARDO-BROWN D. (1990). «Experienced teachers' planning practices: A US survey ". Journal of Education for Teaching, vol. 16, $\mathrm{n}^{\circ} 1$, p. 57-71.

SAUSSEZ F. \& LESSARD C. (2009). «Entre orthodoxie et pluralisme, les enjeux de l'éducation basée sur la preuve ». Revue française de pédagogie, $\mathrm{n}^{\circ} 168$, p. 111-136.

SCHIFTER D. (1995). "Teachers' changing conceptions of the nature of mathematics: Enactment in the classroom ". In B. Nelson, Inquiry and the development of teaching: Issues in the transformation of mathematics teaching. Newton: Center for the development of teaching, p. 17-25.

SCHMIDT W. \& KENNEDY M. (1990). Teachers' and teacher candidates' beliefs about subject matter and about teaching responsibilities. Research report. East Lansing: Michigan State University. Disponible sur
Internet à l'adresse: <http://www.eric.ed.gov/PDFS/ ED320902.pdf> (consulté le 28 septembre 2010).

SCHOMMER M. (1990). «Effects of beliefs about the nature of knowledge on comprehension ". Journal of Educational Psychology, vol. 82, $\mathrm{n}^{\circ} 3$, p. 498-504.

SCHÖN D. (1994). Le praticien réflexif. Montréal : Éd. Logiques.

SHULMAN L. (1987). "Knowledge and teaching: foundations of the new reform ". Harvard Educational Review, vol. $57, \mathrm{n}^{\circ} 1$, p. 1-22.

SIEGLER R. (1999). Intelligences et développement de l'enfant. Variations, évolution, modalités. Bruxelles: De Boeck.

SIGEL I. (1985). «A conceptual analysis of beliefs ». In I. Sigel, Parental beliefsystems: The psychological consequences for children. Hillsdale : Erlbaum, p. 345-371.

SIMON M. \& MAZZA W. (1993). «From learning mathematics to teaching mathematics: A case study of a prospective teacher in a reform-oriented program ". Communication présentée à l'Annual meeting of the North American chapter of the International group for the psychology of mathematics education, Monterey.

SINATRA G. \& KARDASH C. (2004). «Teacher candidates' epistemological beliefs, dispositions, and views on teaching as persuasion ". Contemporary Educational Psychology, vol. 29, $\mathrm{n}^{\circ}$ 4, p. 483-489.

SMITH D. \& NEALE D. (1989). «The construction of subject matter knowledge in primary science teaching ». Teaching and Teacher Education, vol. 5, $\mathrm{n}^{\circ}$ 1, p. 1-20.

SPARKS G. (1988). "Teachers' attitude toward change and subsequent improvements in classroom teaching". Journal of Educational Psychology, vol. 80, $\mathrm{n}^{\circ} 1$, p. 111117.

SPARKS G. \& LOUCKS-HORSLEY S. (1990). "Models of staff development ». In W. Houston, Handbook of Research on Teacher Education. New York : MacMillan, p. 234-250.

STALLINGS J. (1989). « School achievement effects and staff development: What are some critical factors? " Communication présentée à l'Annual meeting of the American educational research association, San Francisco.

STALLINGS J. \& KRASAVAGE E. (1986). «Program implementation and student achievement in a four-year Madeleine Hunter Follow-Through project ». Elementary School Journal, vol. 87, $\mathrm{n}^{\circ} 2$, p. 117-138.

STAUB F. \& STERN E. (2002). "The nature of teachers' pedagogical content beliefs matters for students' achievement gains: Quasi-experimental evidence from elementary mathematics ". Journal of Educational Psychology, vol. 94, n², p. 344-355.

STIPEK D., GIVVIN K., SALMON J. \& MacGYVERS V. (2001). "Teachers' beliefs and practices related to mathematics instruction". Teaching and Teacher Education, vol. $17, \mathrm{n}^{\circ} 2$, p. 213-226.

STRAUSS S. (1993). "Theories of learning and development for academics and educators ». Educational Psychologist, vol. 28, $n^{\circ} 3$, p. 191-203.

STRAUSS S. (1996). «Confessions of a born-again structuralist». Educational Psychologist, vol. 31, $\mathrm{n}^{\circ} 1$, p. 15-21.

STRAUSS S. \& SHILONY T. (1994). « Teachers' models of children's minds and learning ». In L. Hirschfeld \& S. Gelman, Mapping the mind: Domains pecificity in 
cognition and culture. Cambridge : Cambridge University Press, p. 455-473.

TABACHNICK B. \& ZEICHNER K. (1984). « The impact of the student teaching on the development of teacher perspectives ". Journal of Teacher Education, vol. $35, n^{\circ} 6$, p. 28-36.

TARDIF M. \& LESSARD C. (1999). Le travail des enseignants au quotidien. Bruxelles : De Boeck.

TERHART E. (2001). Lehrerberuf und Lehrerbildung. Weinheim : Beltz.

THOMPSON A. (1992). «Teachers' beliefs and conceptions: A synthesis of the research ". In D. Grows, Handbook of research on mathematics teaching and learning. New York: MacMillan, p. 127-146.

TICKLE L. (1991). «New teachers and the emotions of learning teaching ". Cambridge Journal of Education, vol. 21, $n^{\circ}$ 3, p. 319-329.

TILEMA H. \& KNOL W. (1997). «Promoting student teacher learning through conceptual change or direct instruction ". Teaching and teacher Education, vol. 13, $\mathrm{n}^{\circ} 6$ p. 579-595.

TOMANEK D. (1994). «A case of dilemmas: Exploring my assumptions about teaching science ". Science Education, vol. $78, n^{\circ} 5$, p. 399-414.

TVERSKY A. \& KAHNEMAN D. (1974). « Judgment under uncertainty: Heuristics and biases ». Science, vol. 185, $n^{\circ} 4157$, p. 1124-1130.

VAN DRIEL J., BULTE A. \& VERLOOP N. (2007). «The relationship between teacher's general beliefs about teaching and learning and their domain specific curricular beliefs ". Learning and Instruction, vol. 17, $\mathrm{n}^{\circ} 2$, p. 156-171.

VAN FLEET A. (1979). « Learning to teach: The cultural transmission analogy ». Journal of Thought, vol. 14, $\mathrm{n}^{\circ} 4$, p. 281-290.

VAUSE A. (2009). «Les croyances et connaissances des enseignants à propos de l'acte d'enseigner: vers un cadre d'analyse ". Les Cahiers de recherche en éducation et formation, $\mathrm{n}^{\circ} 66$.

VEENMAN S. (1984). "Perceived problems of beginning teachers ". Review of Educational Research, vol. 54, $\mathrm{n}^{\circ} 2$, p. 143-177.

VERLOOP N., VAN DRIEL J. \& MEIJER P. (2001). «Teacher knowledge and the knowledge base of teaching ». International Journal of Educational Research, vol. $35, n^{\circ} 5$, p. 441-461.

WAGNER A. (2003). "Conflicts in consciousness: Imperative cognitions can lead to knots in thinking ". In M. Kompf \& P. Denicolo, Teacher Thinking twenty years on: Revisiting persisting problems and advances in education. Lisse : Swets \& Zeitlinger B.V., p. 197-208.

WANLIN P. \& CRAHAY M. (soumis). « La pensée des enseignants pendant l'interaction en classe". Éducation et didactique.

WANLIN P., LAFONTAINE D. \& CRAHAY M. (soumis). « Les dilemmes dans la double cyclicité de la pensée planificatrice des enseignants". Revue suisse des sciences de l'éducation.
WEINSTEIN C. (1988). «Preservice teachers' expectations about the first year of teaching ". Teaching and Teacher Education, vol. 4, n¹, p. 31-40.

WEINSTEIN C. (1989). "Teacher education students' preconceptions of teaching ". Journal of Teacher Education, vol. 40, $\mathrm{n}^{\circ} 2$, p. 53-60.

WEINSTEIN R., MADISON S. \& KUKLINSKI M. (1995). "Raising expectations in schooling: Obstacles and opportunities for change". American Educational Research Journal, vol. 32, $\mathrm{n}^{\circ} 1$, p. 121-159.

WILCOX S., SHRAM P., LAPPAN G. \& LANIER P. (1991). The role of a learning community in changing preservice teachers' knowledge. East Lansing: Michigan State University.

WILSON S. (1990). "The secret garden of teacher education ». Phi Delta Kappa, vol. 72, n 3, p. 204-209.

WILSON W. \& KATAYANI M. (1968). "Intergroup attitudes and strategies in games between opponents of the same or a different race ". Journal of personnality and social psychology, vol. 9, $\mathrm{n}^{\circ} 1$, p. 24-30.

WINDSCHITL M. (2002). "Framing constructivism in practice as the negotiation of dilemmas: An analysis of the conceptual, pedagogical, cultural, and political challenges facing teachers ". Review of Educational Research, vol. 72, $\mathrm{n}^{\circ} 2$, p. 131-175.

WINITZKY N. (1992). "Structure and process in thinking about classroom management: An exploratory study of prospective teachers ». Teaching and Teacher Education, vol. 8, $\mathrm{n}^{\circ} 1$, p. 1-14.

WINITZKY N. \& KAUFCHAK D. (1997). «Constructivism in teacher education: Applying cognitive theory to teacher learning ". In V. Richardson, Constructivist education: Building news understandings. Londres : Falmer Press, p. 59-83.

WOOLFOLK HOY A. \& BURKE SPERO R. (2005). " Changes in teacher efficacy during the early years of teaching: $A$ Comparison of four measures ". Teaching and Teacher Education, vol. 21, $\mathrm{n}^{\circ} 4$, p. 343-356.

WOOLFOLK HOY A. \& MURPHY P. (2001). « Teaching educational psychology to the implicit mind ". In B. Torff \& R. Sternberg, Understanding and teaching the intuitive mind. Mahwah : Lawrence Erlbaum, p. 145-186.

WOOLFOLK HOY A., DAVIS H. \& PAPE S. (2006). " Teachers' knowledge and beliefs". In P. Alexander \& P. Winne, Handbook of educational psychology [2 ${ }^{\mathrm{e}}$ éd.]. Mahwah : Lawrence Erlbaum, p. 715-737.

YILDIRIM A. (2003). " Instructional planning in a centralized school system: Lessons of a study among primary school teachers in Turkey ". International Review of Education, vol. 49, $\mathrm{n}^{\circ}$ 5, p. 525-543.

ZAHORIK J. (1990). "Stability and flexibility in teaching". Teaching and Teacher Education, vol. 6, $\mathrm{n}^{\circ} 1$, p. 69-80.

ZEICHNER K., TABACHNIK R. \& DENSMORE K. (1987). "Individual, institutional, and cultural influences on the development of the teachers' craft knowledge". In J. Calderhead, Exploring teacher thinking. Londres: Cassell, p. 21-59. 
Annexe 1. - Études ayant trait à l'effet des programmes de formation initiale sur l'évolution des croyances et connaissances des futurs enseignants

\begin{tabular}{|c|c|c|c|c|}
\hline \multirow[b]{2}{*}{$\begin{array}{l}\text { Cible } \\
\text { du changement }\end{array}$} & \multicolumn{4}{|c|}{ Conclusion tirée par les auteurs } \\
\hline & Pas d'évolution & $\begin{array}{l}\text { Évolution } \\
\text { (d'au moins } \\
\text { certains } \\
\text { étudiants) }\end{array}$ & $\begin{array}{l}\text { Changements } \\
\text { timides } \\
\text { après } \\
\text { une phase } \\
\text { de résistance }\end{array}$ & $\begin{array}{c}\text { Certaines } \\
\text { catégories } \\
\text { de croyances } \\
\text { évoluent, } \\
\text { d'autres pas }\end{array}$ \\
\hline $\begin{array}{l}\text { Adhésion au } \\
\text { constructivisme }\end{array}$ & Olson (1993) & $\begin{array}{c}\text { Hollingsworth } \\
\text { (1989), } \\
\text { Ammon (1991), } \\
\text { Levin \& Ammon } \\
(1992)\end{array}$ & & \\
\hline $\begin{array}{l}\text { Croyances } \\
\text { pédagogiques } \\
\text { générales }\end{array}$ & $\begin{array}{c}\text { Saban, } \\
\text { Kocbeker } \\
\text { \& Saban (2007) }\end{array}$ & $\begin{array}{c}\text { Jones \& Vesilind } \\
\text { (1996) }\end{array}$ & & $\begin{array}{c}\text { Feinman-Nemser, } \\
\text { McDiarmid, } \\
\text { Melnick et al. } \\
\text { (1989) }\end{array}$ \\
\hline $\begin{array}{l}\text { Démarche } \\
\text { réflexive }\end{array}$ & $\begin{array}{c}\text { Munro (1993), } \\
\text { Tickle (1991) }\end{array}$ & $\begin{array}{c}\text { Korthagen } \\
(1988), \\
\text { Bolin (1990) }\end{array}$ & $\begin{array}{c}\text { Philip, } \\
\text { Armstrong } \\
\text { \& Bezuk (1993) }\end{array}$ & $\begin{array}{l}\text { Korthagen } \\
\text { (1988) }\end{array}$ \\
\hline $\begin{array}{l}\text { Enseignement } \\
\text { constructiviste } \\
\text { des } \\
\text { mathématiques }\end{array}$ & $\begin{array}{c}\text { Wilcox, Shram, } \\
\text { Lappan et al. } \\
(1991)\end{array}$ & & Civil (1996) & \\
\hline $\begin{array}{l}\text { Éducation } \\
\text { multiculturelle }\end{array}$ & $\begin{array}{c}\text { McDiarmid } \\
\text { (1992) }\end{array}$ & & & \\
\hline Redoublement & $\begin{array}{c}\text { Crahay \& Ory } \\
\qquad(2006)\end{array}$ & & & \\
\hline $\begin{array}{l}\text { Identité } \\
\text { professionnelle }\end{array}$ & & & & $\begin{array}{l}\text { Woolfoolk Hoy } \\
\text { \& Burke Spero } \\
\text { (2005) }\end{array}$ \\
\hline $\begin{array}{l}\text { Conduite } \\
\text { de la classe } \\
\text { Hétérogénéité } \\
\text { des élèves }\end{array}$ & $\begin{array}{c}\text { Zeichner, } \\
\text { Tabachnick } \\
\text { \& Densmore } \\
\text { (1987) } \\
\text { Programme } \\
\text { TELT }\end{array}$ & $\begin{array}{c}\text { Winitzky (1992), } \\
\text { Winitzky } \\
\text { \& Kaufchak } \\
(1997)\end{array}$ & & \\
\hline $\begin{array}{l}\text { Planification } \\
\text { et conduites } \\
\text { d'enseignement }\end{array}$ & $\begin{array}{c}\text { Tilema \& Knol } \\
\text { (1997) }\end{array}$ & $\begin{array}{c}\text { Philip, } \\
\text { Armstrong } \\
\text { \& Bezuk (1993) }\end{array}$ & & \\
\hline
\end{tabular}




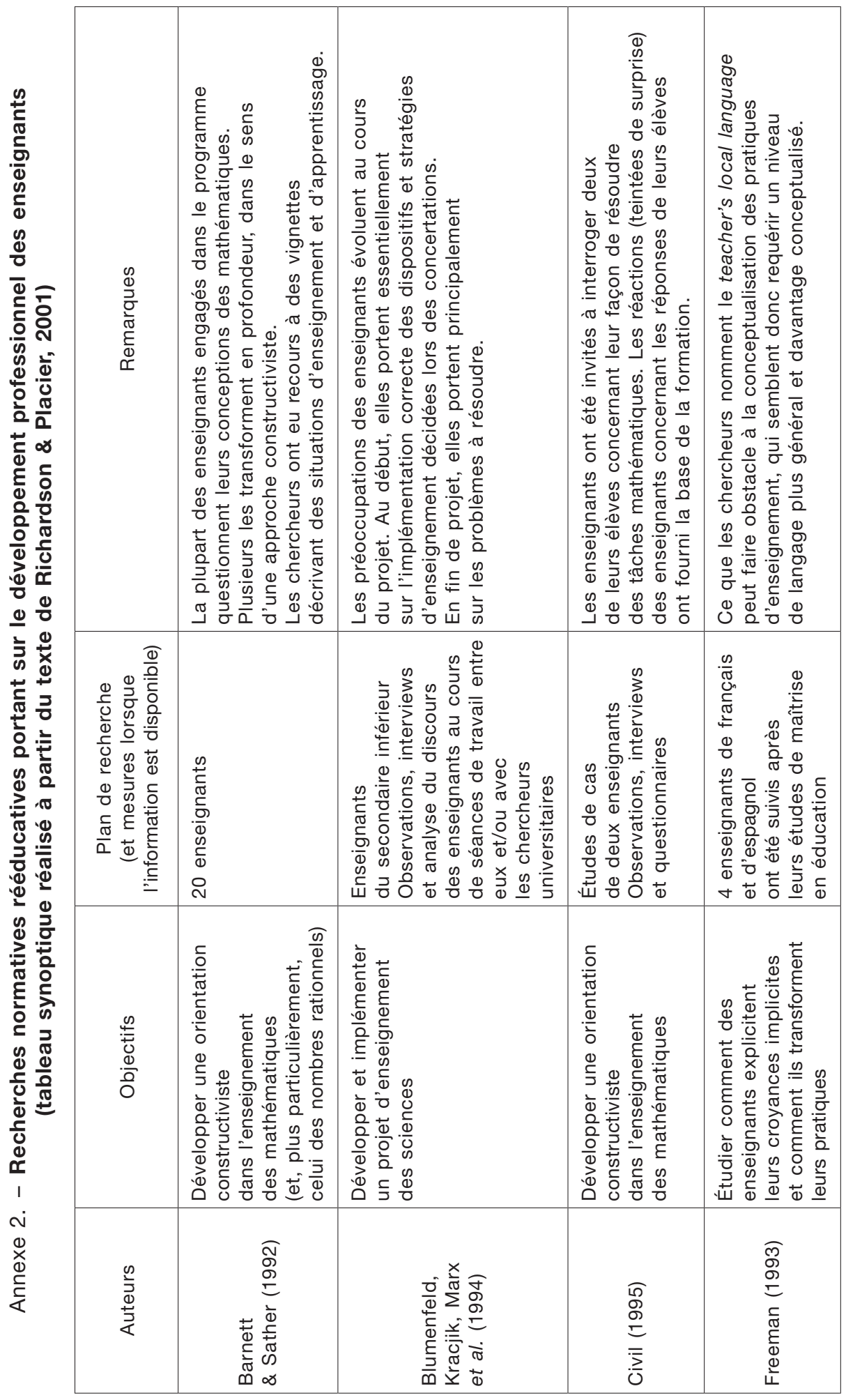




\begin{tabular}{|c|c|c|c|c|c|}
\hline 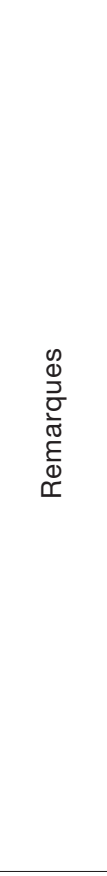 & 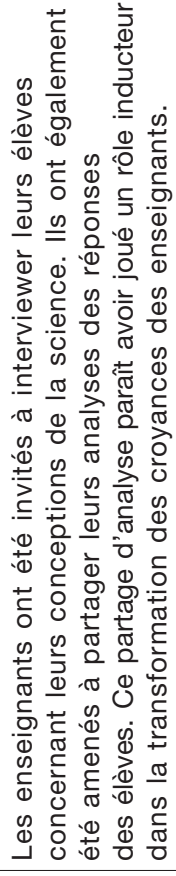 & 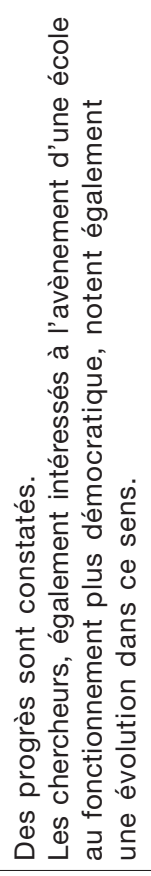 & 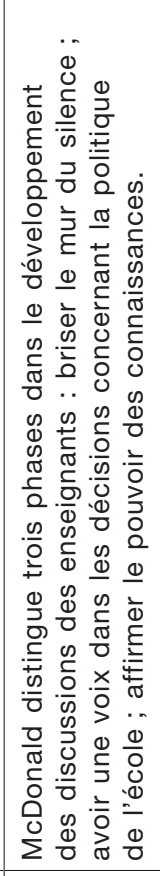 & 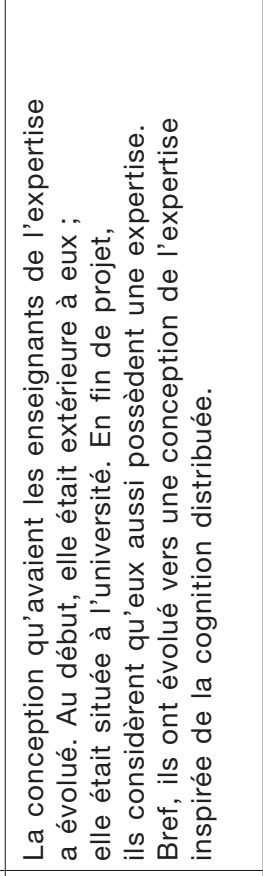 & 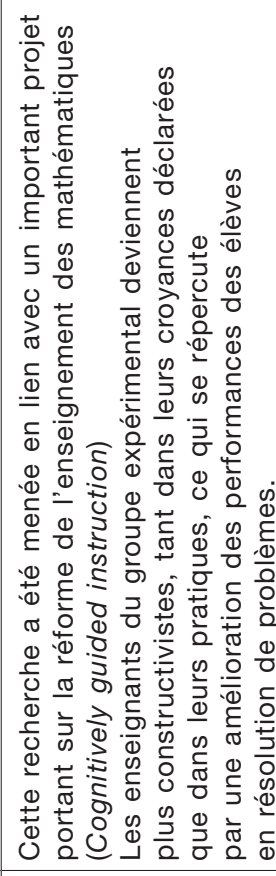 \\
\hline 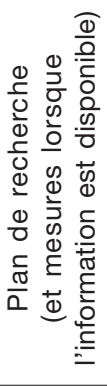 & 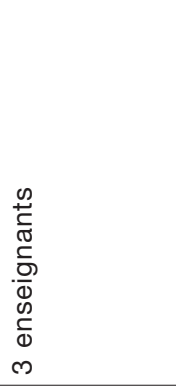 & 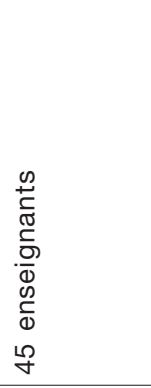 & 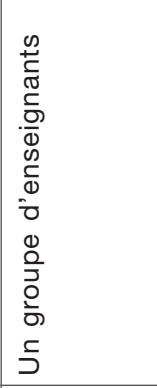 & 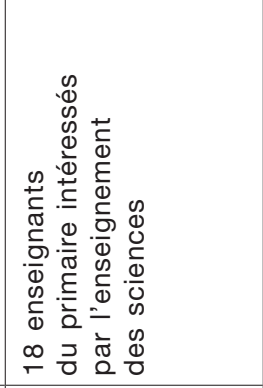 & 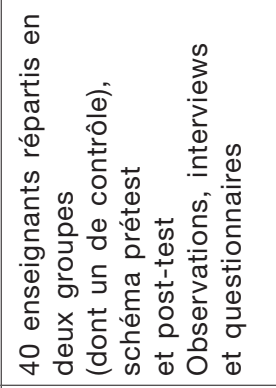 \\
\hline $\begin{array}{l}\stackrel{0}{ \pm} \\
\stackrel{0}{0} \\
\stackrel{\oplus}{0} \\
\stackrel{0}{0}\end{array}$ & 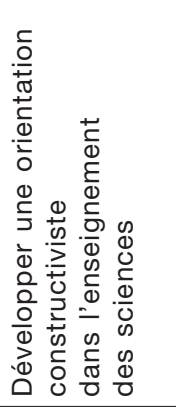 & 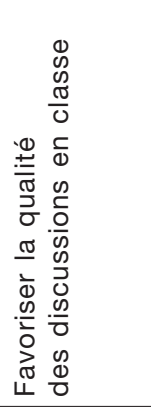 & 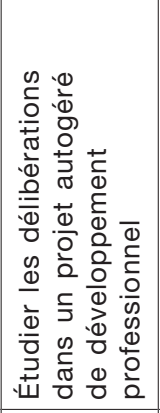 & 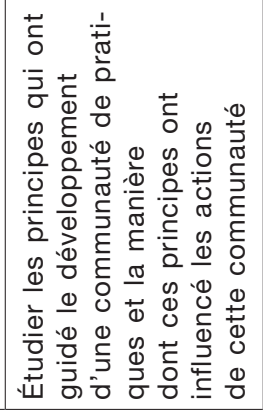 & 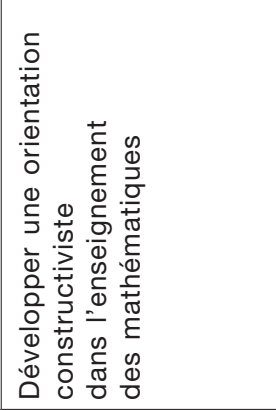 \\
\hline 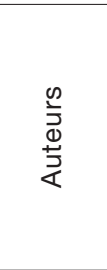 & 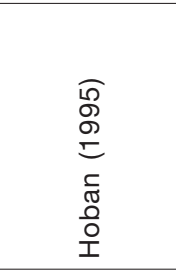 & 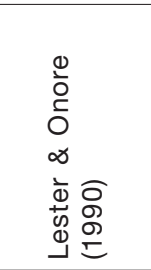 & 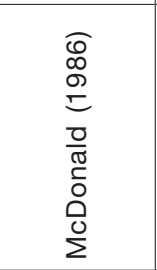 & 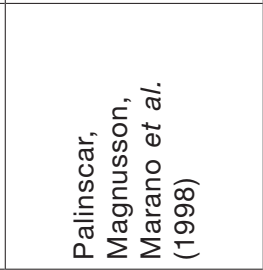 & 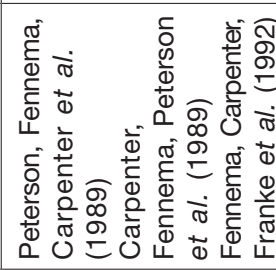 \\
\hline
\end{tabular}




\begin{tabular}{|c|c|c|c|}
\hline 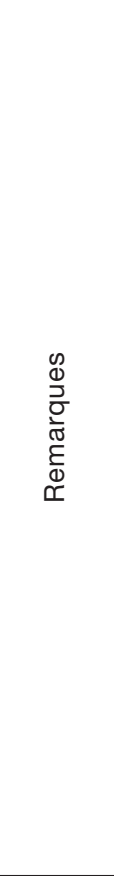 & 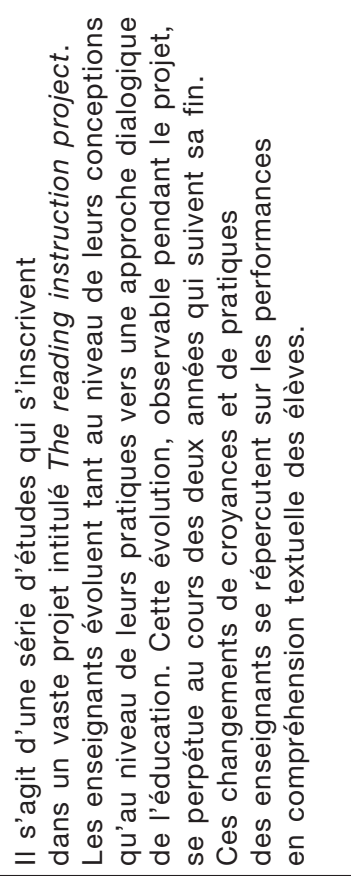 & 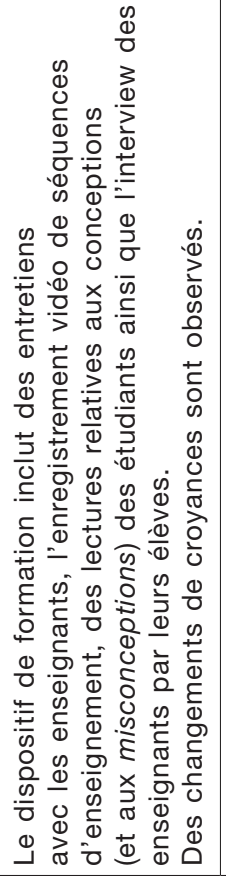 & 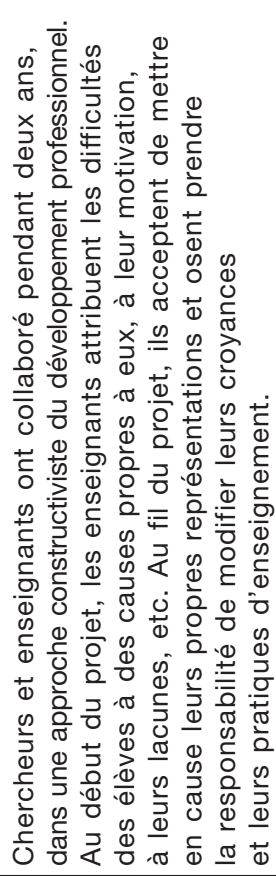 \\
\hline 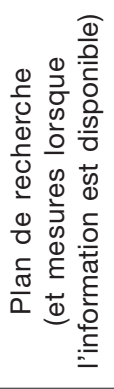 & 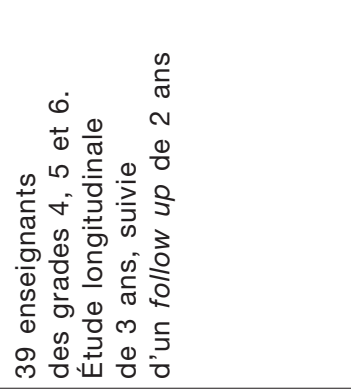 & & 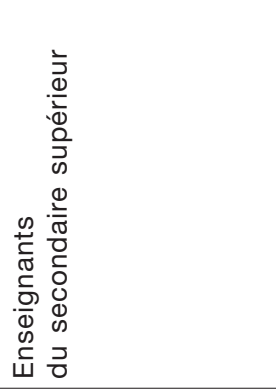 \\
\hline 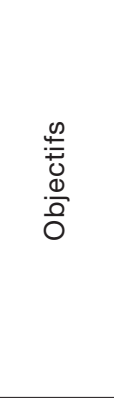 & 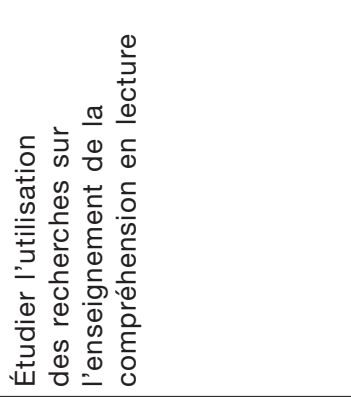 & 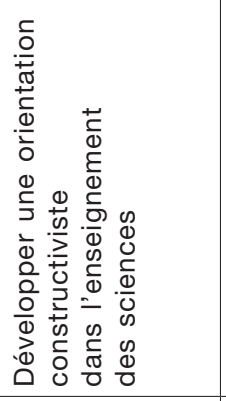 & 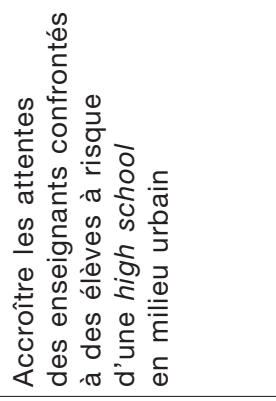 \\
\hline 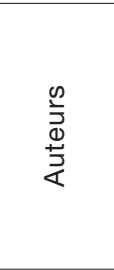 & 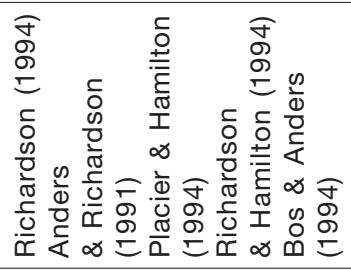 & 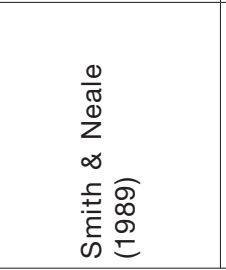 & 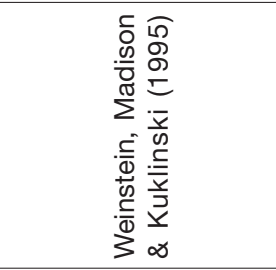 \\
\hline
\end{tabular}

